by Yongfeng Zhu ${ }^{l}$, Bo Chen ${ }^{l}$, Xin X $u^{2}$, Tian Qiu ${ }^{l}$, Fang $A n^{3}$

\title{
A new geological map of the western Junggar, north Xinjiang (NW China): Implications for Paleoenvironmental reconstruction
}

\author{
1 The Key Laboratory of Orogenic Belts and Crustal Evolution, Ministry of Education; School of Earth and Space Science, Peking University, \\ Beijing 100871, China.E-mail: yfzhu@pku.edu.cn \\ 2 Xinjiang Resource and Environment Research Center, Urumqi 830000, China \\ 3 Department of Geology, Northwest University, Xi'An 710054, China
}

Recently, new geochronological data and geological observations including the discovery of garnet amphibolite, Ordovician micro-fossils, unconformable contact between the ophiolitic mélange related flysch and the Devonian to Early Carboniferous terrestrial volcanosedimentary strata provide critical evidences for Paleoenvironmental reconstruction in the western Junggar (Xinjiang, China). Two different geological layers could be clearly identified: the Early Paleozoic metamorphic terrane mainly consists of ophiolitic mélange and flysch, and the Late Paleozoic volcano-sedimentary units lying on the ophiolitic mélange and flysch. Both of these layers were intruded by granitic to dioritic magma during the Late Carboniferous to Early Permian period. Based on these data, a new geological map for the western Junggar has been compiled, which is essential not only for better understanding of the geological evolution but also for exploration of mineral resources.

\section{Introduction}

The Central Asian Paleozoic Ocean (CAPO) is represented by a complex collage of fragments of oceanic crust (ophiolites), oceanic islands, ancient micro-continents and arc terranes. It is interpreted as having formed by the accretion of island arcs and intervening oceans probably started from the Cambrian-Ordovician to Devonian (e.g., Zhu and Ogasawara, 2002; He et al., 2004; Windley et al., 2007; Xiao et al., 2008; Kröner et al., 2008; Han et al., 2011; Choulet et al., 2012; Wilhem et al., 2012; Zhao and He, 2012). Its multi-stage geological evolution makes this huge area a unique and complicated large-scale metal province (Heinhorst et al., 2000; Kurchavov et al., 2002; Yakubuchuk, 2004). Locating on the central part of the CAPO, the western Junggar is characterized by linear zones of Paleozoic ophiolites, volcano-sedimentary rocks, and intermediate to granitic intrusions (see Fig. 1).

The Tangbale, Darbut-Sartohay, and Baijiantan-Baikouquan ophiolitic belts, as the major components of the western Junggar, have been studied for interpreting the Paleozoic subduction-accretion processes. The Tangbale ophiolite mélange consists of radiolarian chert, pillow lava, metagabbro, serpentinite, harzburgite and lherzolite. Consistent with stratigraphical records, radiometric dating of metagabbro (489Ma, Zhang et al, 1993) and titanite in leucogabbro (502 Ma, Kwon et al., 1989) suggested that the Tangbale ophiolite mélange was formed at Late Cambrian. The time for other ophiolitic mélanges was not well constrained. For example, the wall-rocks of the Sartohay ophiolitic mélange contain Early to Middle Devonian fossils (Zhu et al., 1987; Wei et al., 1987), while the low-grade metamorphic gabbro samples were dated to be $395 \mathrm{Ma}(\mathrm{Sm}-\mathrm{Nd}$ isochrons, Zhang and Huang, 1992) and 391Ma (zircon U-Pb age, $\mathrm{Gu}$ et al., 2009). Previous studies demonstrated that different rockunits in the Darbut-Sartohay ophiolitic mélanges reflect their different origins. For example, basalts with geochemical characteristics similar to N-MORB, E-MORB and OIB randomly distribute in the Sartohay region (Yang et al., 2000; Lei et al., 2008; Yang et al., 2012). Both metabasites with prehnite-pumpellyite metamorphic phase (Zhang et al., 2001) and garnet amphibolite (Zhu et al., 2008) were found in the Sartohay ophiolitic mélange. The Darbut-Sartohay ophiolitic mélanges were studied since 1980s, while the newly identified BaijiantanBaikouquan ophiolitic belt (Xu et al., 2006; He et al., 2007) needs more attention. The rock-units with different ages and metamorphic phases in these ophiolitic mélanges are not well-constrained. The newly uncovered geological data are conflicting with the previously proposed geological models. For example, the widespread Early Carboniferous volcano-sedimentary rocks in western Junggar, filling in the post-orogenic basin and occurring as molasse in most cases (Zhu et al., 2013), were mis-understood as island arc system in geological literatures. This is critical for tectonic reconstruction of the Kazakhstan-Junggar plate as well as the geological evolution of CAPO. To solve the above problems, more detailed field geological work and re-interpretation of present geological data are necessary. Recently, increase of geological data could illustrate a completely new version for geology evolution in the western Junggar. This paper displays a newly compiled geological map (see Figs. 1, 2) based on new data and the old geological map (BGMRXUA, 1993; He et al., 2004). Several key evidences are described in the following text.

\section{Darbut-Sartohay region}

The Darbut fault is a major sinistral north-eastern to eastern trending zone, which separates a sedimentary package from an 


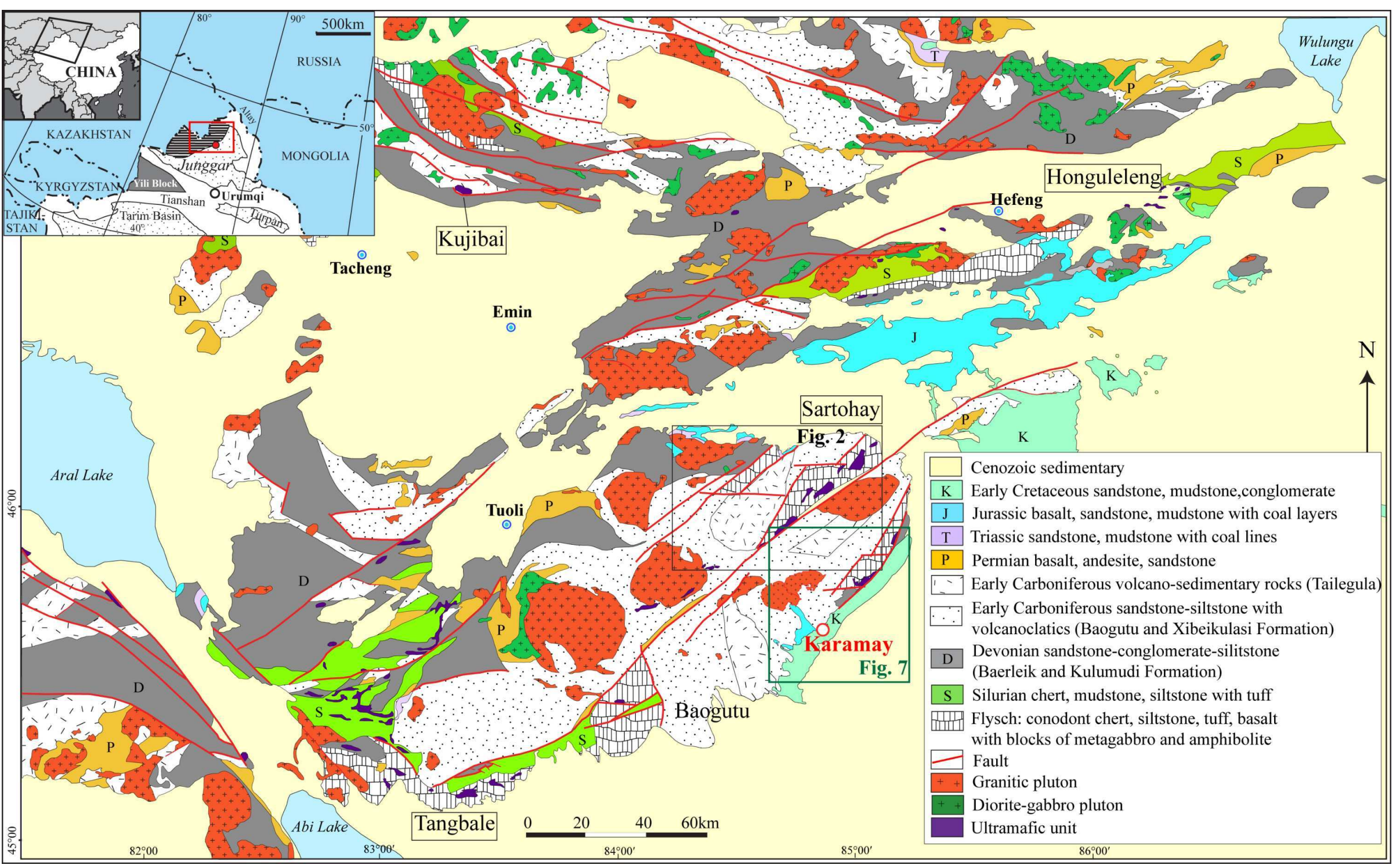

Figure 1. The newly compiled geological map of the western Junggar. 


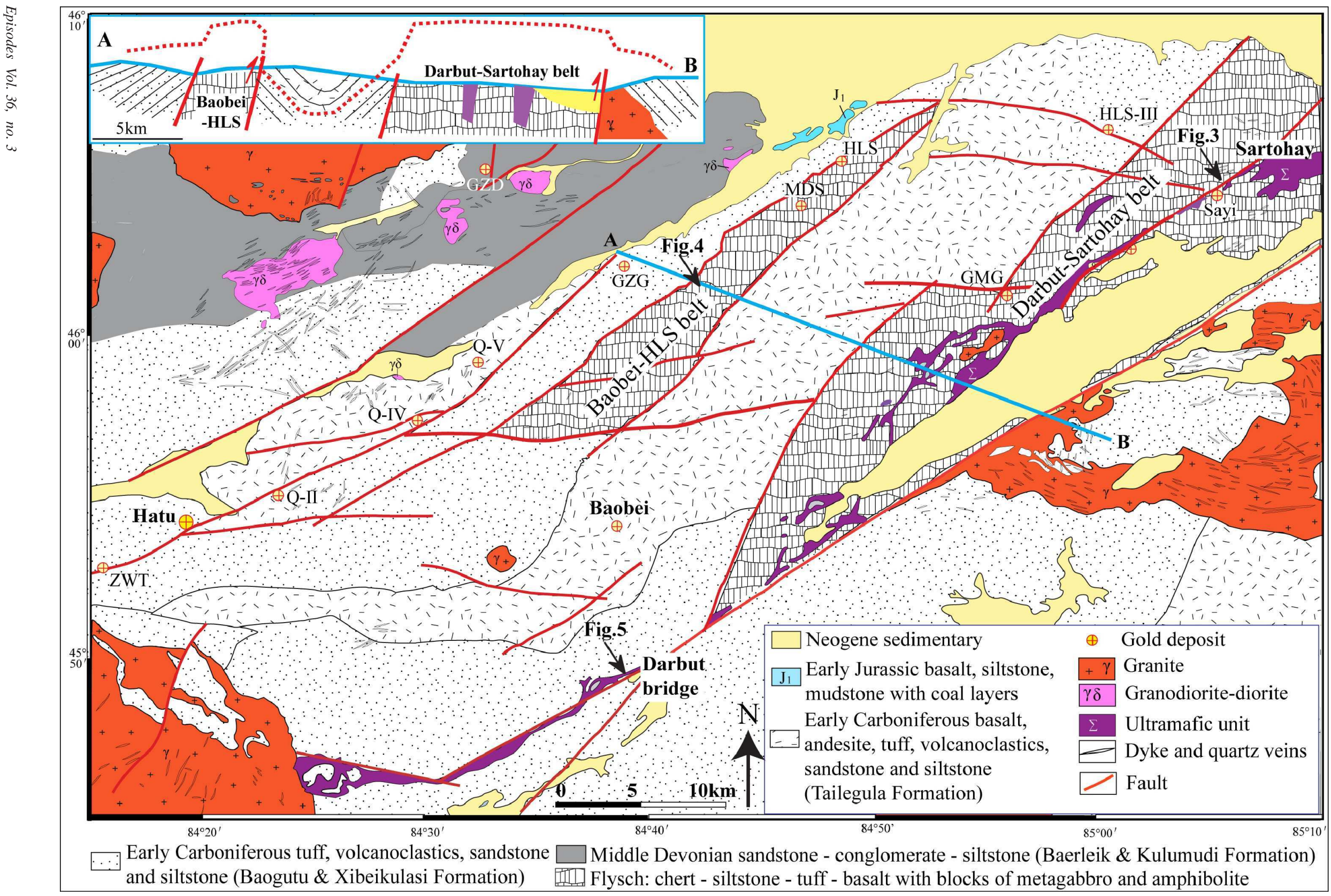

Figure 2. Geological map for the Hatu - Baobei-Sartohay region and a geological section across the Baobei-HLS and Darbut-Sartohay flysch-ophiolitic mélange zones (insert, dashed line indicates the possible positions of the Early Carboniferous volcano-sedimentary strata and the eroded parts of the flysch-ophiolitic mélange). 
ophiolitic mélange containing a chaotic assemblage of blocks of strongly deformed serpentinite, metagabbro, chert and basalts (Feng, 1986; Bai et al, 1995; Chen and Zhu, 2008). Flysch, mainly consisting of chert and siltstone-mudstone-tuff, lies on the ophiolitic mélange. The layers in flysch, generally 5 to $9 \mathrm{~cm}$ in thickness, might be turnover in many cases. In the case as shown in Figs. 3a-b, flysch with normal stratigraphic position lies on basalt and metagabbro. All these rocks are strongly deformed and showing close-spaced cleavage with direction nearly to EW. Magnesitite and fuchsite magnesitite with serpentinite relics, occurring at the lower part of the Sartohay ophiolitic mélange (Fig. 3a), generally shows shearing deformation (Fig. 3c). Sulfide-bearing quartz veins in magnesitite are major target for gold exploration (Qiu and Zhu, 2012). Serpentinite with chromitite lenses occasionally occurs at the stratigraphic bottom of the Sartohay opiolitic mélange (Zhou et al., 2001; Tang and Zhu, 2010). The serpentinite is covered by the Devonian sandstone and conglomerate unconformably (Fig. 3d). The breccias in conglomerate are mainly chert-siltstone. This sandstone - conglomerate does not undergo deformation, while serpentinite was strongly deformed. Flysch, consisting mainly of chert and siltstone with high-angle layers, contacts with flatangle strata of the Early Carboniferous volcanoclastics unconformably (Figs. 3a, e, f).

The Baobei-HLS zone (see Fig. 2 for location), locating on the north of the Darbut-Sartohay zone, consists of chert, siltstone and tuff with basalt locally in the Baobei-Huilvshan region (Fig. 4). The stratigraphic layers of chert and siltstone-tuff are nearly perpendicular and extending NE-direction. Basaltic pillows were cemented by chert in some cases (Fig. 4b). The boundary between siltstone layer and tuff layer is uneven (Fig. 4c). The strata of chert - siltstone - tuff is stable in most cases and it is covered by the Devonian sandstone conglomerate unconformably.

The ophiolitic mélange near the Darbut bridge (Fig. 5a), consisting mainly of serpentinite, basalt, and small blocks of metagabbro, contacts with the Lower Carboniferous tuff, siltstone, and volcanoclastics via fault. The profile section shows a dome structure (Fig. 5b): ultramafic rocks were elevated in core part, where that basalt and metagabbro were mostly eroded. Metagabbro blocks in serpentinite matrix are mainly composed of hydrous garnet

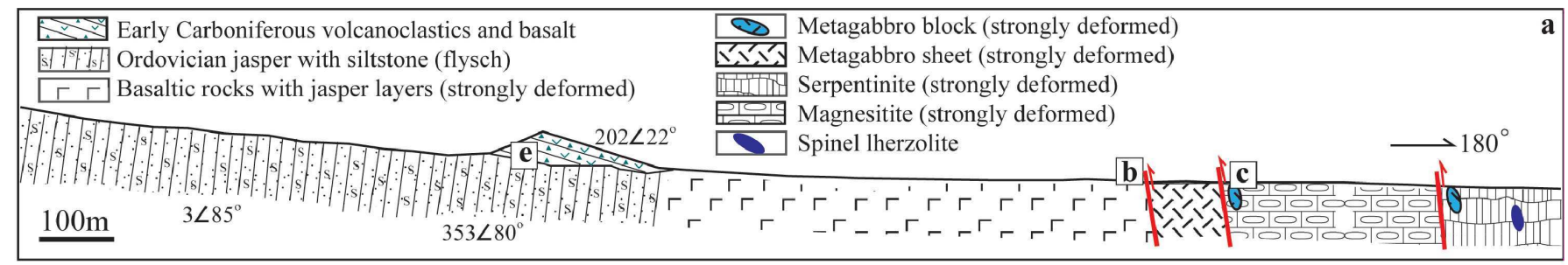
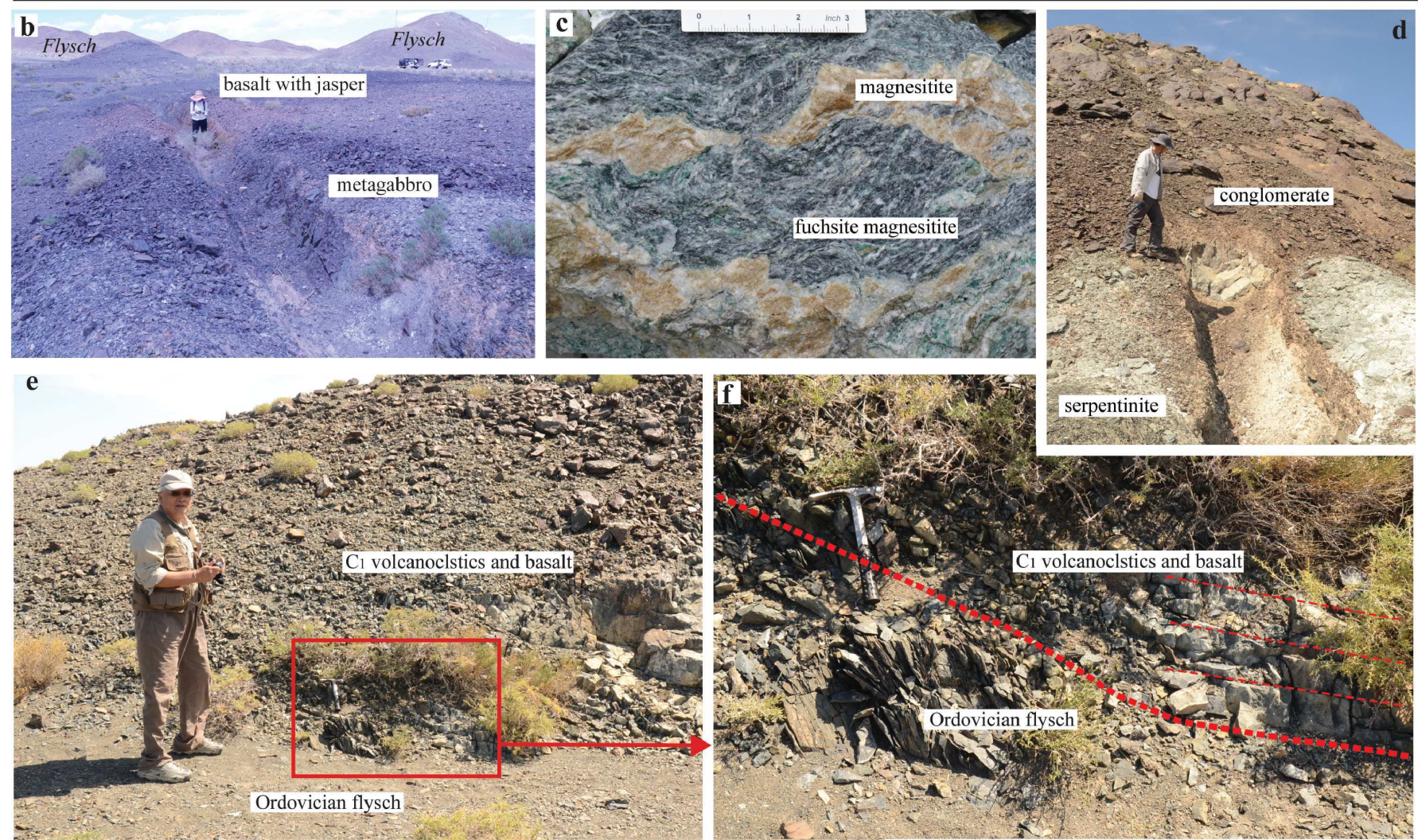

Figure 3. (a) Geological section of the Sartohay ophiolitic mélange (see Fig. 2 for location); (b) A trench exposing the stratigraphic sequence of the ophiolitic mélange. The hills on north are flysch. All these units are strongly deformed; (c) Magnesitite and fuchsite magnesitite, containing Cr-spinel and locating under metagabbro, show shearing deformation; (d) Devonian conglomerate and sandstone lying on the ophiolitic mélange; (e-f) Early Carboniferous volcanoclastics with basalt lie on the ophiolitic mélange unconformably. Note: the chert-siltstone with perpendicular bands has been strongly deformed, while the strata of volcanoclastics do not undergo deformation. 

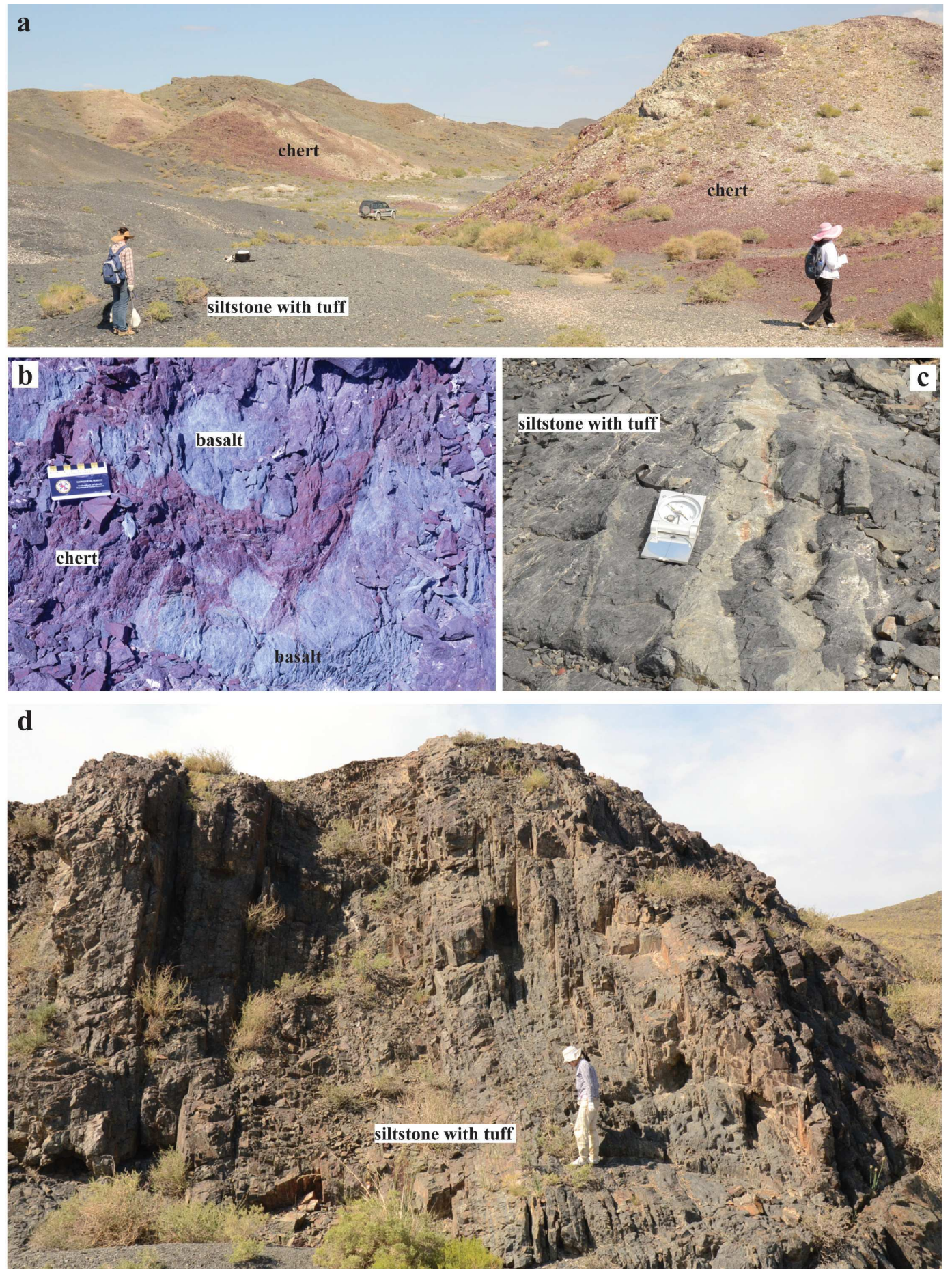

Figure 4. (a) Photos showing chert and siltstone with tuff strata in the Baobei-HLS zone, locally see the basalt was cemented by chert (b); $(c-d)$ Photos showing the perpendicular strata consisting mainly of siltstone and tuff in the Baobei-HLS zone.

(grossularite) and clinopyroxene (see Figs. 5c). The original texture was destroyed, while the assemblage of clinopyroxene and garnet with feldspar pseudomorph still preserves gabbro texture. Feldspar was broken down into albite + zoisite with grossularite locally. Chromite contains inclusion consisting of hornblende and grossularite occasionally (see Fig. 5d). Late-stage amphibole, chlorite, and epidote are widespread in these units.

Zircons were separated from metagabbro for SHRIMP dating. A zircon cathodoluminescence image is showing in Fig. 6a. This zircon has a complex texture with a core, a mantle showing sector-zoning pattern, and a discontinuous rim. The rim was formed during metamorphism. The SHRIMP analyses are mainly performed on igneous zircons with $\mathrm{Th} / \mathrm{U}$ ratio of $0.44-0.57$. Apart from one relatively younger age (358Ma), other six $\mathrm{U}-\mathrm{Pb}$ ages give a weighted mean age of $426.0 \pm 5.8 \mathrm{Ma}(\mathrm{MSWD}=0.87$, Fig. $6 \mathrm{~b}$ ). This age represents the crystallization time of gabbro, and thus implies that the Paleo-ocean floor spreading occurred before the middle Silurian period. The younger age with extremely low $\mathrm{Th} / \mathrm{U}$ ratio $(358 \mathrm{Ma}$, $\mathrm{Th} / \mathrm{U}=0.06$ ), obviously differing from other data, might be a mixture between the igneous mantle and its metamorphic rim. Two detrital 

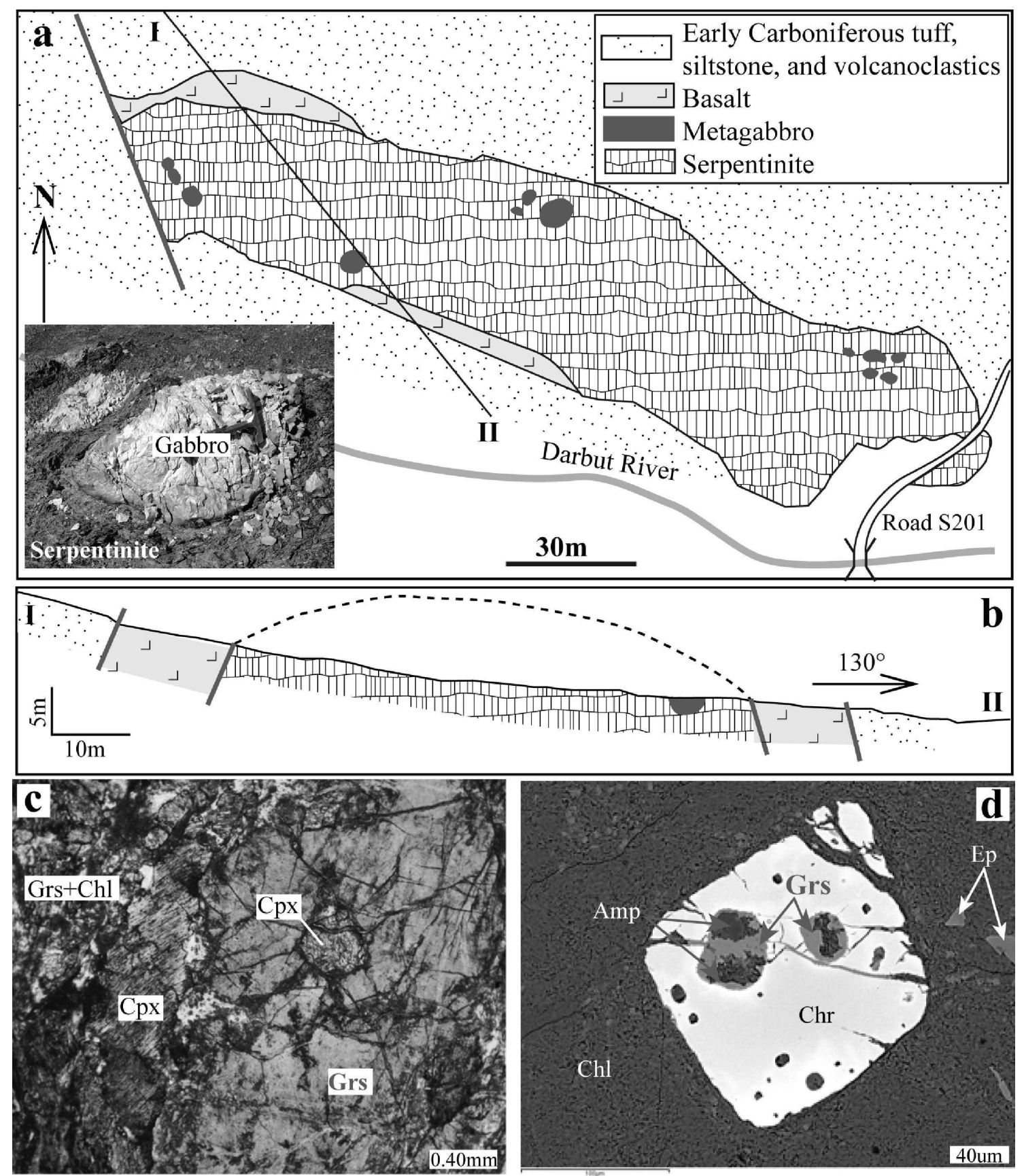

Figure 5. (a-b) A simplified geological map for the outcrop and geological section (see Fig. 2 for location) near the Darbut bridge. Dashed line indicates possible position of the eroded part of the flysch-ophiolitic mélange. Insert photo showing an outcrop of metagabbro blocks in serpentinite; (c-d) Microphotographs showing the mineral assemblages of metagabbro. Ab-albite, Amp - amphibole, Chl - chlorite, Cpx-clinopyroxene, Grs - garnet, Mt - magnetite, Zo-zoisite.

zircon cores give extremely old $\mathrm{U}-\mathrm{Pb}$ ages of $2327 \mathrm{Ma}(\mathrm{Th} / \mathrm{U}$ of 0.62 for spot -3.1$)$ and $1830 \mathrm{Ma}(\mathrm{Th} / \mathrm{U}$ of 0.03 for spot -9.1 , see Table 1$)$. Both of them might belong to relics of continental crust, which have been involved in ophiolitic melanges similar to the detrital zircons in Karamay OIB-type pillow basalt (Zhu et al., 2007) and in metagabbro from the Kujibai ophiolitic mélange (Zhu and Xu, 2006).

\section{Baijiantan-Baikouquan region}

The Baijiantan-Baikouquan ophiolitic belts (Fig. 7a) were identified recently (Xu et al., 2006; He et al., 2007; Zhu et al., 2008).
The Baikouquan ophiolitic mélange is mainly composed of serpentinite with lherzolite lenses, blocks of metagabbro and amphibolite. Marble is rare and basalt occurs as matrix. Although the ophiolitic mélange was strongly weathered, some fresh rock samples still could be collected in the top of some small hills (see Figs. 7b, c). Basaltic rocks lie on serpentinite (see Fig. 7b). Both basalt and serpentinite have undergone strong deformation. The Devonian to Early Carboniferous volcanoclastics cover ophiolitic mélange and flysch (see the section in Fig. 7d).

Differing from the blocks of lherzolite and metagabbro scattered in matrix randomly, blocks containing amphibolite and garnet 


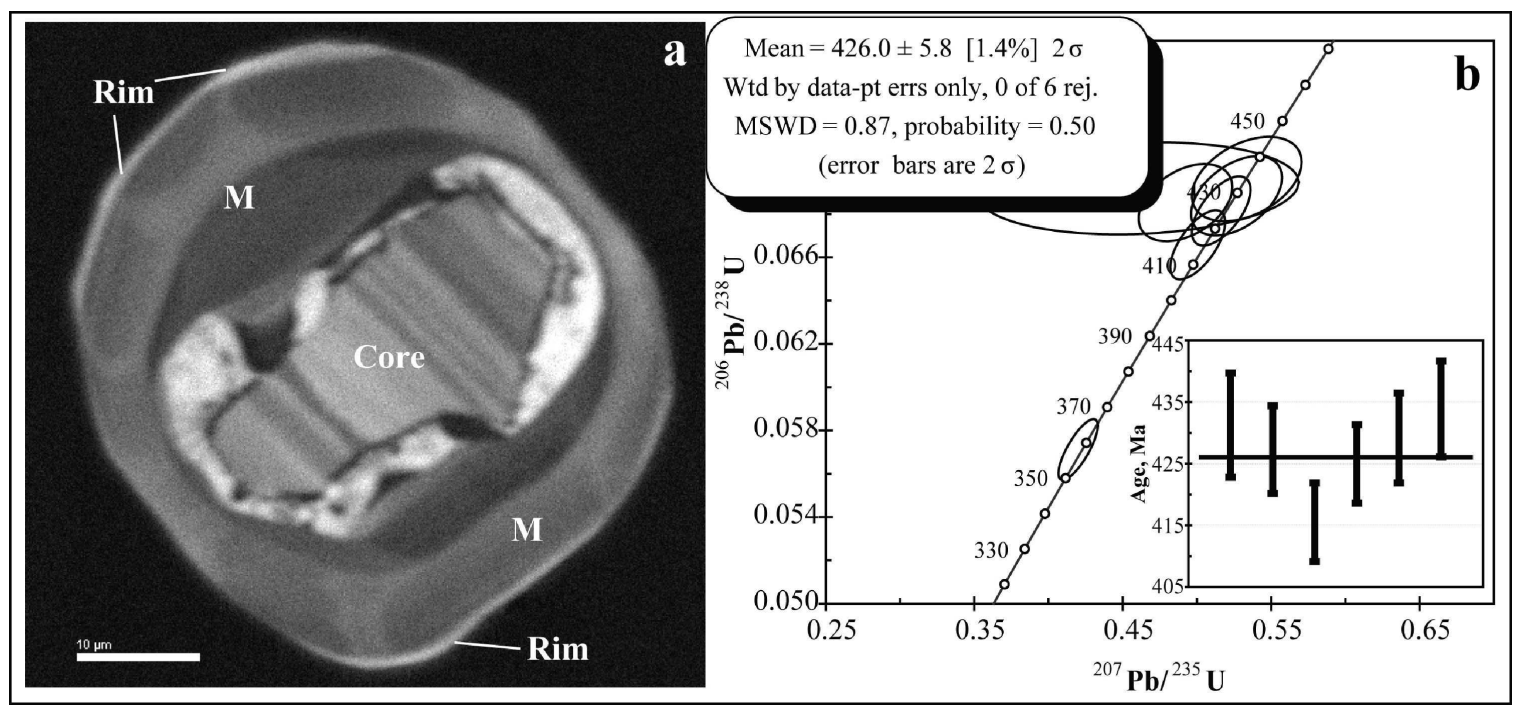

Figure 6. The cathodoluminescence image of a zircon from metagabbro (a) and zircon SHRIMP dating results (b). Scale bar is 10 mm in (a). Detrital zircon core with U-Pb apparent ages of 1830-2367Ma are much older than the igneous zircon mantle (M).
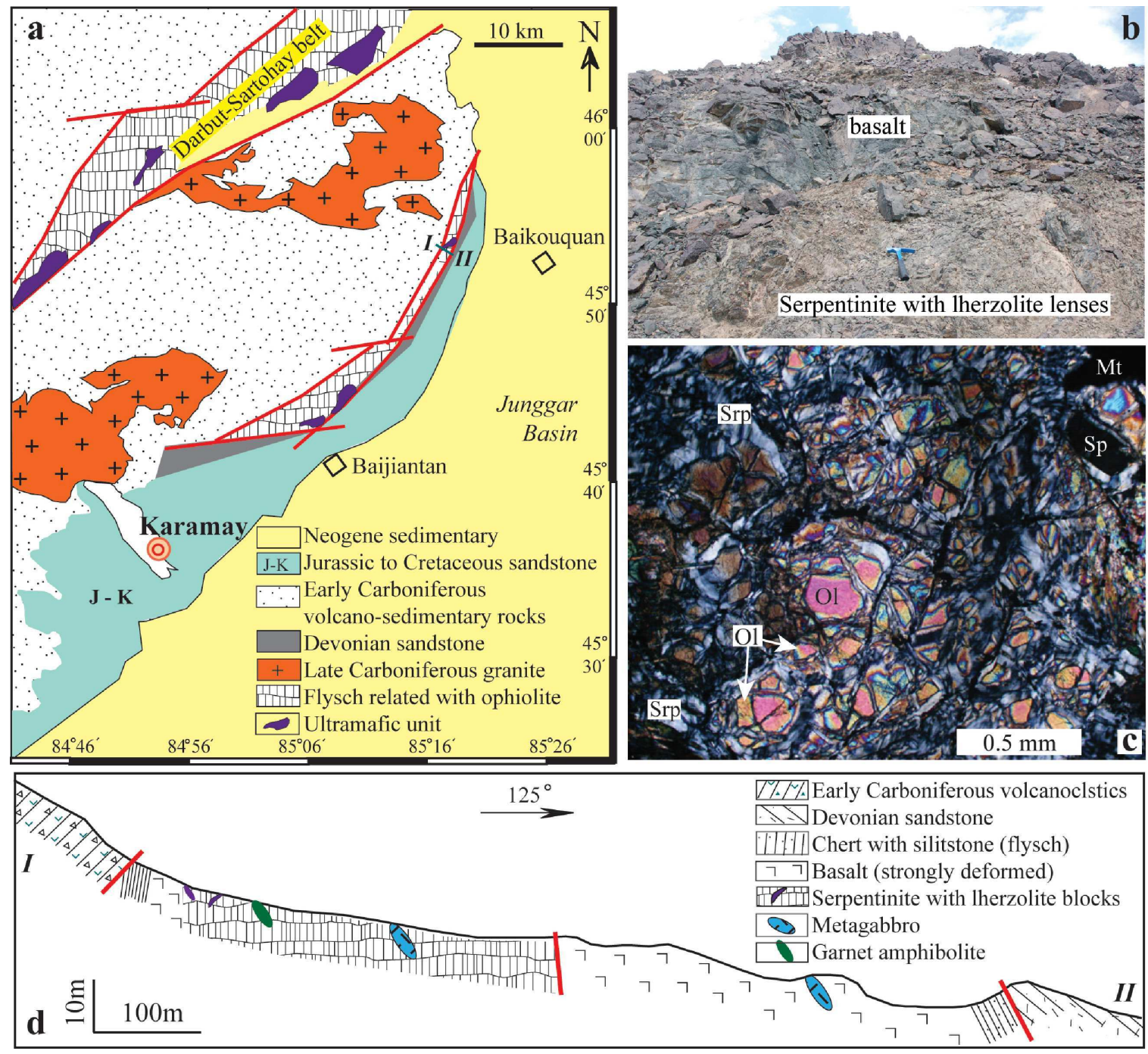

Figure 7. (a) Simplified geological map showing the Darbut-Sartohay and Beijiantan-Baikouquan ophiolitic mélanges; (b) Basalt lies on serpentinite with lherzolite lenses; (c) Microphotograph showing the mineral assemblage in lherzolite; (d) A geological section across the Baikouquan ophiolitic mélange showing blocks of metagabbro, garnet amphibolite, serpentinite in basaltic matrix. These units were overlied by Devonian sandstone and Early Carboniferous volcanoclastics. Mt - magnetite, Ol-olivine, Sp - spinel, Srp - serpentine. 
Table 1 SHRIMP dating results for zircons separated from different rocks in western Junggar (analytical method: zircons were hand picked under a binocular microscope, and cathodoluminescence images obtained using a CAMECA SX-50 microprobe. Zircons were dated using the SHRIMP II installed at the Beijing SHRIMP Center, data correction was based on Williams (1998))

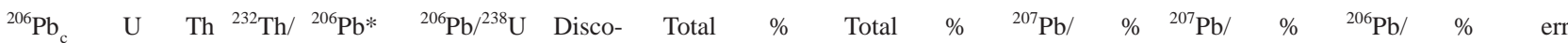

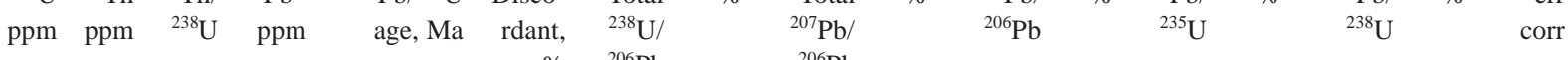

$\% \quad{ }^{206} \mathrm{~Pb} \quad{ }^{206} \mathrm{~Pb}$

\section{DB19}

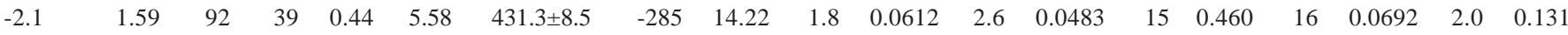

$\begin{array}{lllllllllllllllllll}-3.1 & 0.00 & 272 & 165 & 0.62 & 100 & 2327 * \pm 7.4 & 1 & 2.33 & 1.6 & 0.148 & 0.4 & 0.148 & 0.43 & 8.76 & 1.6 & 0.429 & 1.6 & 0.965\end{array}$

$\begin{array}{llllllllllllllllllll}-5.1 & 0.94 & 163 & 70 & 0.44 & 9.71 & 427.3 \pm 7.1 & -47 & 14.46 & 1.7 & 0.0597 & 2.1 & 0.0521 & 3.9 & 0.492 & 4.2 & 0.0685 & 1.7 & 0.406\end{array}$

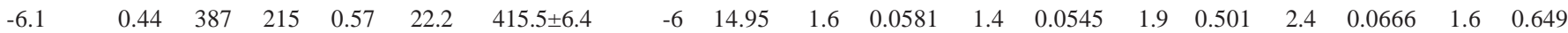

$\begin{array}{lllllllllllllllllll}-7.1 & 0.21 & 856 & 444 & 0.54 & 50.2 & 425.0 \pm 6.4 & -4 & 14.64 & 1.5 & 0.0567 & 1.0 & 0.0550 & 2.0 & 0.516 & 2.5 & 0.0681 & 1.5 & 0.613 \\ -1.2 & 0.13 & 194 & 92 & 0.49 & 11.5 & 429.5 & \end{array}$

$\begin{array}{lllllllllllllllllll}-1.2 & 0.13 & 194 & 92 & 0.49 & 11.5 & 429.2 \pm 7.3 & 1 & 14.51 & 1.7 & 0.0565 & 2.1 & 0.0555 & 3.4 & 0.527 & 3.8 & 0.0688 & 1.8 & 0.458\end{array}$

$\begin{array}{lllllllllllllllllll}-8.1 & 0.06 & 1162 & 64 & 0.06 & 57.1 & 358.1 \pm 5.7 & -5 & 17.50 & 1.6 & 0.0538 & 0.9 & 0.0533 & 1.3 & 0.420 & 2.1 & 0.0571 & 1.6 & 0.782\end{array}$

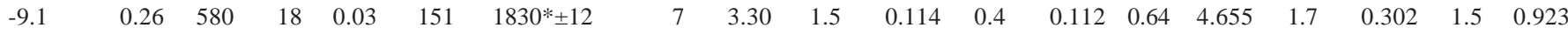

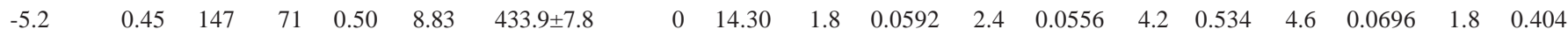

\section{HT-QII}

$\begin{array}{lllllllllllllllllll}-5-1.1 & 4.66 & 172 & 156 & 0.94 & 8.35 & 338.9 \pm 7.4 & -233 & 17.66 & 2.0 & 0.0858 & 4.0 & 0.0481 & 18 & 0.358 & 18 & 0.0540 & 2.2 & 0.125\end{array}$

$\begin{array}{llllllllllllllllllll}-5-2.1 & 4.02 & 190 & 122 & 0.67 & 8.95 & 331.2 \pm 7.1 & 32 & 18.20 & 2.0 & 0.0892 & 4.7 & 0.0569 & 16 & 0.414 & 16 & 0.0527 & 2.2 & 0.139\end{array}$

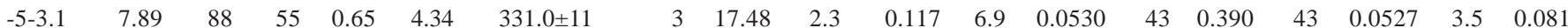

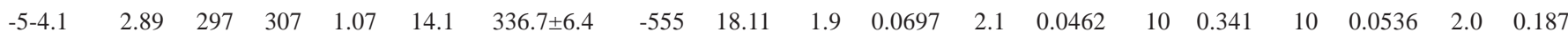

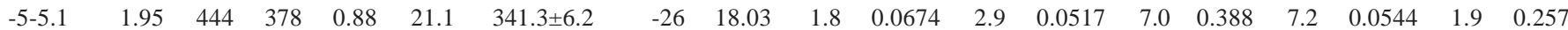

$\begin{array}{llllllllllllllllllll}-5-6.1 & 6.91 & 148 & 74 & 0.52 & 7.18 & 330.3 \pm 7.7 & 173 & 17.71 & 2.1 & 0.0951 & 4.9 & 0.0380 & 29 & 0.279 & 30 & 0.0526 & 2.4 & 0.081\end{array}$

$\begin{array}{lllllllllllllllllll}-5-7.1 & 2.20 & 289 & 158 & 0.57 & 13.5 & 333.0 \pm 7.9 & 24 & 18.45 & 2.3 & 0.0733 & 2.8 & 0.0557 & 12 & 0.407 & 12 & 0.0530 & 2.4 & 0.205\end{array}$

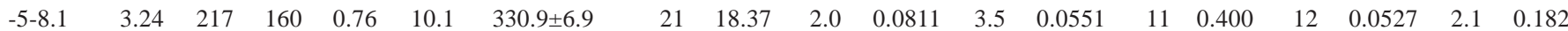

$\begin{array}{lllllllllllllllllll}-5-9.1 & 3.48 & 276 & 135 & 0.51 & 13.1 & 335.4 \pm 6.8 & 529 & 18.07 & 1.9 & 0.0729 & 2.5 & 0.0446 & 16 & 0.328 & 16 & 0.0534 & 2.1 & 0.126\end{array}$

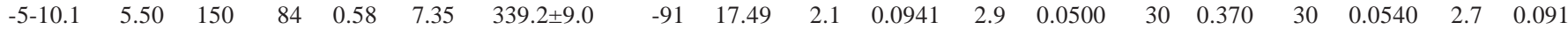

\section{BB10}

$-1.1$

$\begin{array}{rrrrrrr}-3.1 & 1.86 & 118 & 110 & 0.96 & 5.35 & 327.1 \pm 3.9 \\ -113 & 93 & 0.86 & 5.50 & 349.1 \pm 4.4\end{array}$

$\begin{array}{lllllll}-4.1 & 1.53 & 195 & 134 & 0.71 & 8.90 & 328.3 \pm 3.3\end{array}$

$\begin{array}{lllllll}-5.1 & 0.68 & 200 & 132 & 0.68 & 9.10 & 329.9 \pm 3.1\end{array}$

$\begin{array}{lllllll}-6.1 & 1.66 & 95 & 39 & 0.43 & 4.36 & 329.2 \pm 4.3\end{array}$

$\begin{array}{lllllll}-7.1 & 0.79 & 179 & 113 & 0.65 & 8.06 & 327.4 \pm 3.3\end{array}$

$\begin{array}{lllllll}-8.1 & 0.76 & 94 & 65 & 0.71 & 4.28 & 329.3 \pm 4.3\end{array}$

$\begin{array}{lllllll}-9.1 & 0.26 & 191 & 127 & 0.68 & 8.51 & 324.5 \pm 3.1\end{array}$

$\begin{array}{lllllll}-10.1 & 1.90 & 409 & 231 & 0.58 & 18.3 & 321.6 \pm 2.8\end{array}$

$\begin{array}{llllllll}-11.1 & 0.28 & 417 & 242 & 0.60 & 19.5 & 339.8 \pm 2.7\end{array}$

$\begin{array}{lllllll}-12.1 & 0.78 & 174 & 147 & 0.87 & 7.96 & 332.3 \pm 3.3\end{array}$

$\begin{array}{llllllll}-13.1 & 0.40 & 293 & 250 & 0.88 & 13.0 & 323.2 \pm 2.7\end{array}$

$\begin{array}{lllllll}-14.1 & 0.85 & 373 & 414 & 1.15 & 17.2 & 334.5 \pm 2.7\end{array}$

$\begin{array}{lllllll}-15.1 & 0.72 & 286 & 204 & 0.74 & 13.0 & 331.2 \pm 2.8\end{array}$ $\begin{array}{llllllllllll}153 & 18.65 & 1.2 & 0.0629 & 5.2 & 0.0456 & 13 & 0.330 & 13 & 0.0525 & 1.4 & 0.105\end{array}$ $\begin{array}{llllllllllll}23 & 19.01 & 1.2 & 0.0636 & 4.1 & 0.0553 & 6.8 & 0.397 & 6.9 & 0.0521 & 1.2 & 0.175\end{array}$ $\begin{array}{llllllllllll}587 & 17.64 & 1.2 & 0.0599 & 2.7 & 0.0447 & 10 & 0.343 & 10 & 0.0556 & 1.3 & 0.122\end{array}$ $\begin{array}{llllllllllll}-781 & 18.85 & 0.96 & 0.0592 & 2.8 & 0.0468 & 7.4 & 0.337 & 7.5 & 0.0523 & 1.0 & 0.138\end{array}$ $\begin{array}{llllllllllll}4 & 18.91 & 0.95 & 0.0588 & 2.1 & 0.0533 & 3.6 & 0.386 & 3.8 & 0.0525 & 0.97 & 0.257\end{array}$ $\begin{array}{llllllllllll}-633 & 18.77 & 1.3 & 0.0604 & 5.0 & 0.0469 & 11 & 0.339 & 11 & 0.0524 & 1.4 & 0.125\end{array}$ $\begin{array}{llllllllllll}15 & 19.04 & 1.0 & 0.0607 & 4.5 & 0.0543 & 5.9 & 0.390 & 6.0 & 0.0521 & 1.0 & 0.170\end{array}$ $\begin{array}{llllllllllll}34 & 18.94 & 1.3 & 0.0634 & 3.0 & 0.0573 & 6.8 & 0.414 & 6.9 & 0.0524 & 1.3 & 0.195\end{array}$ $\begin{array}{llllllllllll}42 & 19.32 & 0.97 & 0.0608 & 4.3 & 0.0587 & 5.3 & 0.418 & 5.4 & 0.0516 & 0.99 & 0.184\end{array}$ $\begin{array}{llllllllllll}19 & 19.18 & 0.82 & 0.0698 & 1.5 & 0.0546 & 5.1 & 0.385 & 5.2 & 0.0512 & 0.88 & 0.171\end{array}$ $\begin{array}{llllllllllll}28 & 18.43 & 0.81 & 0.0587 & 1.5 & 0.0565 & 2.5 & 0.421 & 2.6 & 0.0541 & 0.82 & 0.310\end{array}$ $\begin{array}{llllllllllll}8 & 18.76 & 1.0 & 0.0600 & 5.3 & 0.0538 & 6.7 & 0.392 & 6.7 & 0.0529 & 1.0 & 0.152\end{array}$ $\begin{array}{llllllllllll}-7 & 19.37 & 0.84 & 0.0556 & 1.8 & 0.0524 & 3.1 & 0.372 & 3.2 & 0.0514 & 0.85 & 0.270\end{array}$ $\begin{array}{llllllllllll}-35 & 18.62 & 0.80 & 0.0580 & 1.6 & 0.0511 & 3.7 & 0.376 & 3.8 & 0.0533 & 0.83 & 0.217\end{array}$ $\begin{array}{llllllllllll}-8 & 18.83 & 0.85 & 0.0583 & 1.8 & 0.0525 & 3.7 & 0.382 & 3.8 & 0.0527 & 0.88 & 0.231\end{array}$

\section{HT12}

$\begin{array}{lllllll}-1.1 & 1.09 & 109 & 74 & 0.70 & 4.19 & 278.6 \pm 7.0\end{array}$

$\begin{array}{llllllll}-2.1 & 0.16 & 290 & 151 & 0.54 & 16.8 & 420.2 \pm 8.4\end{array}$

$\begin{array}{lllllll}-3.1 & 0.15 & 452 & 217 & 0.50 & 28.4 & 454.2 \pm 9.0\end{array}$

$\begin{array}{lllllll}-4.1 & 0.16 & 218 & 137 & 0.65 & 9.98 & 333.5 \pm 7.0\end{array}$

$\begin{array}{llllllll}-5.1 & 1.39 & 257 & 137 & 0.55 & 10.3 & 289.2 \pm 6.2\end{array}$

$\begin{array}{lllllll}-6.1 & 0.50 & 419 & 271 & 0.67 & 18.6 & 322.5 \pm 6.5\end{array}$

$\begin{array}{lllllll}-7.1 & 0.87 & 131 & 73 & 0.58 & 4.90 & 273.1 \pm 5.9\end{array}$

$\begin{array}{lllllll}-8.1 & 0.04 & 341 & 208 & 0.63 & 15.8 & 338.2 \pm 6.8\end{array}$

$\begin{array}{lllllll}-9.1 & 1.51 & 143 & 74 & 0.54 & 5.37 & 271.0 \pm 5.9\end{array}$

$\begin{array}{lllllll}-10.1 & 3.77 & 238 & 148 & 0.64 & 9.37 & 278.1 \pm 6.2\end{array}$

$\begin{array}{lllllll}-11.1 & 27.26 & 196 & 86 & 0.46 & 10.8 & 293.0 \pm 11\end{array}$

$\begin{array}{lllllll}-12.1 & 1.83 & 176 & 89 & 0.52 & 6.59 & 270.7 \pm 6.3\end{array}$

$\begin{array}{lllllll}-13.1 & 0.65 & 129 & 78 & 0.62 & 5.03 & 283.9 \pm 6.2\end{array}$

$\begin{array}{lllllll}-14.1 & 0.58 & 119 & 76 & 0.66 & 4.46 & 272.8 \pm 6.1\end{array}$ $\begin{array}{llllllllllll}340 & 22.40 & 2.2 & 0.0527 & 3.0 & 0.0440 & 24 & 0.267 & 24 & 0.0442 & 2.6 & 0.108\end{array}$ $\begin{array}{llllllllllll}17 & 14.82 & 2.1 & 0.0587 & 1.4 & 0.0575 & 2.4 & 0.534 & 3.1 & 0.0674 & 2.1 & 0.658\end{array}$ $\begin{array}{llllllllllll}9 & 13.68 & 2.0 & 0.0584 & 1.2 & 0.0572 & 2.1 & 0.576 & 3.0 & 0.0730 & 2.1 & 0.692\end{array}$ $\begin{array}{llllllllllll}14 & 18.81 & 2.1 & 0.0558 & 2.0 & 0.0544 & 4.7 & 0.399 & 5.1 & 0.0531 & 2.2 & 0.421\end{array}$ $\begin{array}{llllllllllll}-563 & 21.50 & 2.1 & 0.0581 & 2.1 & 0.0469 & 7.3 & 0.297 & 7.6 & 0.0459 & 2.2 & 0.287\end{array}$ $\begin{array}{llllllllllll}-46 & 19.39 & 2.0 & 0.0546 & 1.4 & 0.0506 & 3.0 & 0.358 & 3.6 & 0.0513 & 2.1 & 0.566\end{array}$ $\begin{array}{llllllllllll}-21 & 22.90 & 2.2 & 0.0577 & 4.1 & 0.0506 & 6.9 & 0.302 & 7.3 & 0.0433 & 2.2 & 0.305\end{array}$ $\begin{array}{llllllllllll}28 & 18.56 & 2.0 & 0.0568 & 1.4 & 0.0564 & 1.9 & 0.419 & 2.8 & 0.0539 & 2.1 & 0.726\end{array}$ $\begin{array}{llllllllllll}373 & 22.94 & 2.2 & 0.0564 & 6.0 & 0.0442 & 12 & 0.262 & 12 & 0.0429 & 2.2 & 0.185\end{array}$ $\begin{array}{llllllllllll}28 & 21.83 & 2.1 & 0.0845 & 3.1 & 0.0543 & 14 & 0.330 & 14 & 0.0441 & 2.3 & 0.163\end{array}$ $\begin{array}{llllllllllll}66 & 15.63 & 2.1 & 0.282 & 3.6 & 0.0670 & 43 & 0.430 & 43 & 0.0465 & 3.8 & 0.089\end{array}$ $\begin{array}{llllllllllll}176 & 22.89 & 2.2 & 0.0548 & 2.5 & 0.0399 & 18 & 0.236 & 19 & 0.0429 & 2.4 & 0.129\end{array}$ $\begin{array}{llllllllllll}-98 & 22.07 & 2.2 & 0.0541 & 2.8 & 0.0489 & 4.0 & 0.304 & 4.5 & 0.0450 & 2.2 & 0.489\end{array}$ $\begin{array}{llllllllllll}-34 & 23.00 & 2.2 & 0.0549 & 3.2 & 0.0502 & 7.7 & 0.299 & 8.0 & 0.0432 & 2.3 & 0.284\end{array}$

J34

$\begin{array}{lllllllllllllllllll}-1.1 & 2.15 & 590 & 536 & 0.94 & 26.5 & 321.7 \pm 5.7 & -120 & 19.13 & 1.8 & 0.0663 & 1.6 & 0.0490 & 6.4 & 0.345 & 6.6 & 0.0512 & 1.8 & 0.275\end{array}$

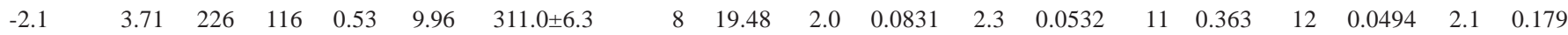

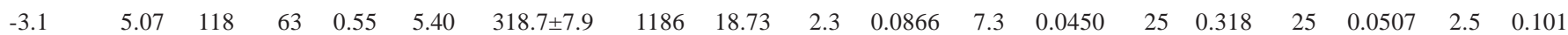
\begin{tabular}{lllllllllllllllllll}
-4.1 & 2.69 & 224 & 97 & 0.45 & 9.76 & $309.9 \pm 6.1$ & 55 & 19.76 & 2.0 & 0.0836 & 3.0 & 0.0623 & 8.0 & 0.423 & 8.3 & 0.0493 & 2.0 & 0.246 \\
\hline
\end{tabular} 
Table 1. Contd...

\begin{tabular}{|c|c|c|c|c|c|c|c|c|c|c|c|c|c|c|c|c|c|c|}
\hline & ${ }^{206} \mathrm{~Pb}_{\mathrm{c}}$ & $\begin{array}{r}\mathrm{U} \\
\mathrm{ppm}\end{array}$ & $\begin{array}{r}\text { Th } \\
\text { ppm }\end{array}$ & $\begin{array}{l}{ }^{232} \mathrm{Th} / \\
{ }^{238} \mathrm{U}\end{array}$ & $\begin{array}{r}{ }^{206} \mathrm{~Pb}^{*} \\
\mathrm{ppm}\end{array}$ & $\begin{array}{r}{ }^{206} \mathrm{~Pb} /{ }^{238} \mathrm{U} \\
\text { age, Ma }\end{array}$ & $\begin{array}{r}\text { Disco- } \\
\text { rdant, } \\
\%\end{array}$ & $\begin{array}{l}\text { Total } \\
{ }^{238} \mathrm{U} / \\
{ }^{206} \mathrm{~Pb}\end{array}$ & $\%$ & $\begin{array}{r}\text { Total } \\
{ }^{207} \mathrm{~Pb} / \\
{ }^{206} \mathrm{~Pb}\end{array}$ & $\%$ & $\begin{array}{l}{ }^{207} \mathrm{~Pb} / \\
{ }^{206} \mathrm{~Pb}\end{array}$ & $\%$ & $\begin{array}{l}{ }^{207} \mathrm{~Pb} / \\
{ }^{235} \mathrm{U}\end{array}$ & $\%$ & ${ }^{206} \mathrm{~Pb} /$ & $\%$ & \\
\hline 5.1 & 4.61 & 259 & 174 & 0.69 & 11.3 & $305.3 \pm 6.9$ & -797 & 19.66 & 2.2 & 0.0840 & 2.3 & 0.0467 & 15 & 0.312 & 15 & 0.0485 & 2.3 & 0.152 \\
\hline 6.1 & 2.97 & 370 & 189 & 0.53 & 16.5 & $16.5 \pm 6.0$ & -59 & 19.28 & 1.9 & 0.0740 & 2.0 & 0.0501 & 8.9 & 0.348 & 9.1 & .0503 & 1.9 & .211 \\
\hline 7.1 & 2.98 & 533 & 335 & 0.65 & 23.2 & $09.5 \pm 5.9$ & 234 & 9.72 & 1.8 & 0.0662 & 1.7 & 0.0419 & 14 & 0.284 & 15 & .0492 & .0 & .13 \\
\hline 8.1 & 3.66 & 353 & 189 & 0.55 & 14.1 & $81.3 \pm 9.2$ & -723 & 21.60 & 3.2 & 0.0763 & 2.6 & 0.0467 & 16 & 0.287 & 16 & .0446 & 3 & .209 \\
\hline 9.1 & 10.19 & 60 & 22 & 0.39 & 2.96 & $5.0 \pm 12$ & 57 & 7.38 & 2.7 & 0.145 & 3.5 & .0650 & 33 & 0.460 & 33 & .0517 & .8 & .113 \\
\hline 10.1 & & 341 & 195 & 0.59 & 13.3 & $5.5 \pm 5.5$ & 11 & 05 & 1.9 & 0.0856 & 3.7 & .0526 & 13 & 0.317 & 13 & .0437 & & .154 \\
\hline 11.1 & & 233 & 146 & 0.65 & 10.8 & $4.3 \pm 6.9$ & 194 & & 0 & & 2.4 & & 20 & & 20 & .0516 & & 0.111 \\
\hline-12.1 & & 169 & 84 & 0.51 & 7.78 & $8.4 \pm 7.1$ & -11 & 1 & 2.0 & & 4.3 & & 19 & & 19 & & & 0.11 \\
\hline 13.1 & & 374 & 196 & 0.54 & 16.7 & $4.3 \pm$ & -9 & & & & 1.9 & & 10 & & 10 & .0500 & & 0.190 \\
\hline 14 & & 190 & 121 & 0.66 & 8.1 & & & & & & & & 21 & & & & & .10 \\
\hline & & & & & & & & & & & & & 14 & & & & & .12 \\
\hline & & & & & & & & & & & & & & & & & & .1 \\
\hline 17. & & 6 & 28 & & 2.8 & & & & & & & & 55 & & & & & .0 \\
\hline 18. & & 198 & 97 & 0.51 & 8.8 & 5.9 & & & & & & 429 & 20 & & & 0497 & & 0.115 \\
\hline 19.1 & & 272 & 117 & 0.44 & 12.0 & 6.0 & -456 & 1 & & & 2.5 & 0.0472 & 6.3 & & 6.6 & .0504 & 9 & 0.296 \\
\hline 20.1 & & 270 & 178 & 0.68 & 11.9 & & 79 & 1 & 1.9 & & 3.1 & 0.0452 & 14 & 06 & 14 & .0491 & 2.0 & 0.142 \\
\hline 21.1 & 4.67 & 268 & 140 & 0.54 & 12.0 & $312.2 \pm 6.3$ & 246 & 19.21 & 1.9 & 0.0802 & 3.3 & 0.0422 & 17 & 0.289 & 17 & 0.0496 & 2.1 & 0.124 \\
\hline \multicolumn{19}{|l|}{$\mathrm{J} 35$} \\
\hline & & & & & & & & & & & & & 56 & & & & & \\
\hline & & & 6 & & & & & & & & & & 37 & & 37 & & & 0.073 \\
\hline & & 145 & 7 & 0.56 & 6.51 & & & & & & 4 & 0.040 & 29 & & & .0488 & .5 & .086 \\
\hline & & 128 & 6 & 0.56 & 5.7 & & & & 2.1 & & 2 & 0.0 & 32 & & 32 & & 6 & .08 \\
\hline .1 & & 129 & 73 & 0.58 & 5.98 & $313.6 \pm$ & 1339 & 18.60 & 2.1 & 0.10 & 4.6 & 0.046 & 29 & 0.3 & 29 & 0.0499 & 2.6 & 0.090 \\
\hline & & 128 & 66 & 0.53 & 5.89 & $309.4 \pm$ & & & 2. & 0.1 & 3.6 & 0.036 & 36 & & 36 & 0492 & 2.6 & 0.072 \\
\hline & & 120 & 65 & 0.56 & 5.53 & $307.9 \pm 8.3$ & 137 & 1870 & 2.3 & 0.103 & 4.0 & 0.053 & 41 & & 42 & 0489 & 2.8 & 0.067 \\
\hline & & 147 & 79 & 0.56 & 6.85 & $323.4 \pm 7.3$ & 53 & 18.44 & 2.1 & 0.103 & 5.9 & 0.062 & 17 & & 17 & & 2.3 & 0.138 \\
\hline & & 122 & 63 & 0.53 & 5.64 & $311.5 \pm 8.2$ & & 18.64 & 2.2 & 0.0938 & 5.9 & 0.030 & 49 & 0.204 & 49 & 0.0495 & 2.7 & 0.055 \\
\hline 10 & & 131 & 70 & 0.55 & 6.14 & $318.3 \pm 8.0$ & & 18.28 & & 0.102 & 5.7 & 0.041 & 33 & 0.286 & 33 & 0.0506 & 2.6 & 0.078 \\
\hline 11.1 & & 152 & 76 & 0.52 & 6.92 & $314.2 \pm 7.3$ & -293 & 18.89 & & 0.0933 & 5.0 & 0.048 & 22 & 0.328 & 23 & 0.0499 & 2.4 & 0.10 \\
\hline & & 142 & 79 & 0.57 & 6.4 & $305.3 \pm$ & & & & & 4. & & 39 & 0.230 & 39 & .0485 & 2.6 & 0.06 \\
\hline & & 146 & 81 & 0.57 & 6.8 & $17.7 \pm$ & 108 & & & & 4 & 0.0 & 28 & & 28 & .0505 & .5 & 0.0 \\
\hline & & 135 & 76 & 0.58 & 6.3 & $314.9 \pm$ & & & & & 3. & & 33 & & 34 & 0.0501 & 2.7 & 0.08 \\
\hline & & & & & & & & & & & & & 38 & & 38 & & 2.6 & 0.0 \\
\hline & & & & & & & & & & & & & 35 & & & & 2.6 & 0 \\
\hline & & 155 & & & & & & & & & & & 27 & & 27 & & 2.4 & 0.089 \\
\hline-18.1 & 7.73 & 102 & 63 & 0.63 & 4.64 & $306.2 \pm 9.6$ & 53 & 18.97 & 2.4 & 0.123 & 7.8 & 0.061 & 33 & 0.410 & 33 & 0.0486 & 3.2 & 0.09 \\
\hline
\end{tabular}

* refers to the ${ }^{207} \mathrm{~Pb} /{ }^{206} \mathrm{~Pb}$ age.

amphibolite distribute along fault only (see Figs. 8a, b). The metamorphic degree for serpentinite, metagabbro, basaltic rocks, and chert (greenschist facies) are relatively lower than that for amphibolite and garnet amphibolite. Amphibolite consists of hornblende, plagioclase, titanite, ilmenite and epidote. Garnet amphibolite consists of hornblende, clinopyroxene (diopsite-augite), garnet, zoisite, quartz, apatite, titanite, ilmenite and rutile. Hornblende was generally replaced by chlorite. Garnet amphibolite does not contain any plagioclase, which decomposed during prograde metamorphism. This implies that garnet amphibolite was formed at relatively high P-T condition (up to eclogite facies) comparing with amphibolite (amphibolite facies). Garnet contains abundant inclusions including quartz, ilmenite, rutile, apatite, and zircon (Figs. 8c-d). Pyrope contents in garnet vary from $10 \mathrm{~mol} . \%$ to $26 \mathrm{~mol} . \%$ (see Fig. 8e), which is similar to the garnet in eclogite coexisting with blueschist (Coleman et al., 1965). Blueschist, dated to be $458 \sim 470 \mathrm{Ma}$ by ${ }^{40} \mathrm{Ar} /{ }^{39} \mathrm{Ar}$ technique (for sodium amphibole, Zhang, 1997), occurs in the Tangbale ophiolitic mélange locating on the SW of the Baijiantan-Baikouquan ophiolitic belt (see Fig. 1 for location). We have suggested that the Baijiantan-Baikouquan ophiolitic belt should be connected with the Tangbale ophiolitic mélange (Zhu et al., 2008).

Locating on the southwest of the Baikouquan region, the Baijiantan ophiolitic mélange consists of serpentinite, spinel lherzolite, olivine pyroxenite, metagabbro, and spinel dolomite marble (see Figs. 9a-b, 10a). Fresh lherzolite lenses consist of olivine, clinopyroxene, orthopyroxene and spinel. Pyroxene, showing exsolution texture with clinopyroxene lamellae in orthopyroxene, and orthopyroxene lamellae in clinopyroxene, generally underwent ductile-shearing deformation. The spinel dolomite marble, containing relics of spinel serpentinite (Fig. 9b), was transformed from serpentinite during metamorphism (Zhu et al., 2008).

As a result of intensive serpentinization, the mélange area is rather elastic and composes a negative landform (see Figs. 9a, 10a). Pillow basalt was cemented by lava and/or by red chert (Fig. 9c). All these units were covered by conglomerate-sandstone with chert breccia. Flysch with conodont-bearing chert has been intruded by the Permian pyroxene diorite dykes (Fig. 10b). He et al. (2007) found abundant conodonts in red chert (jasper, see Fig. 10c), and suggested that the 


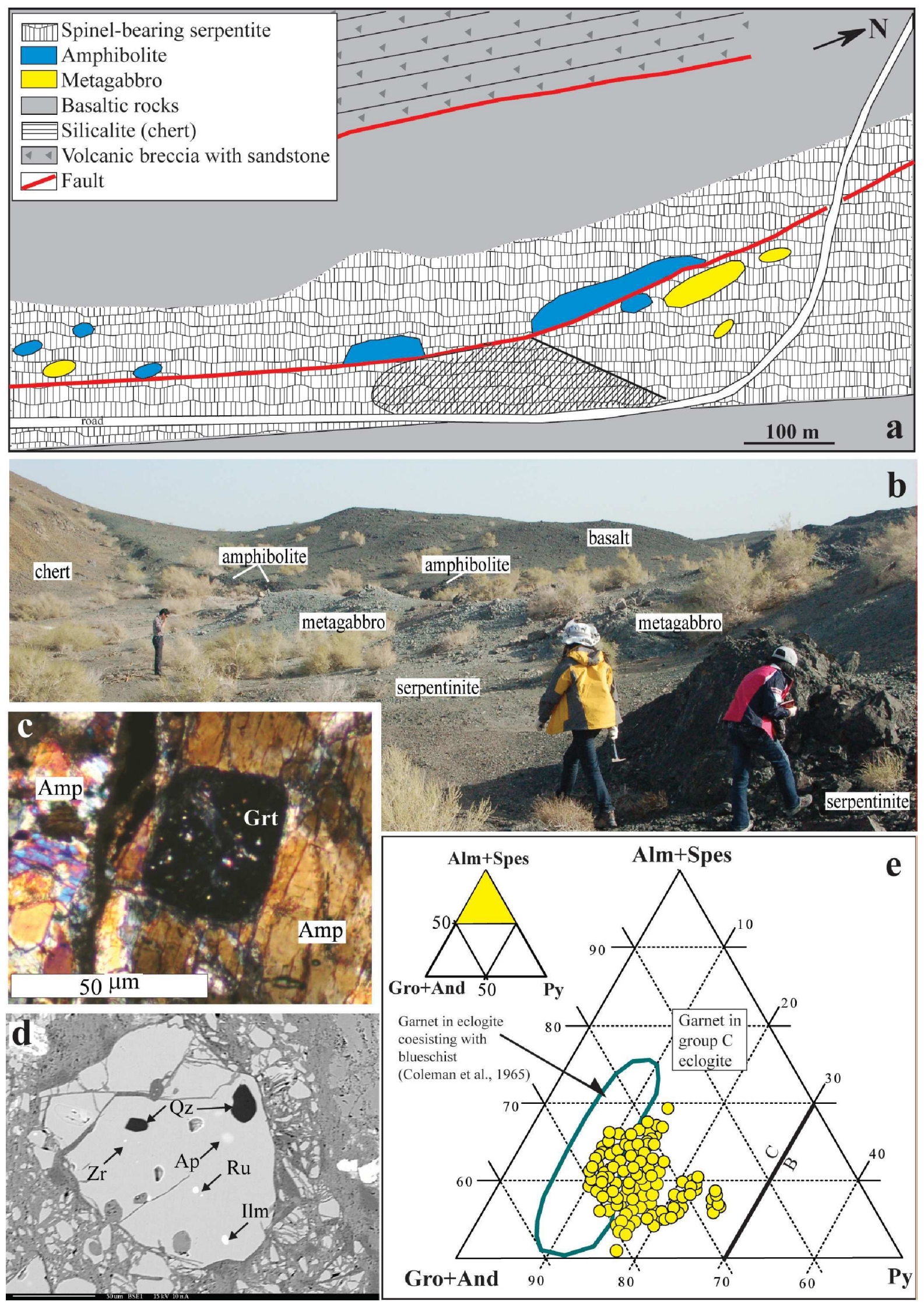

Figure 8. (a) An outcrop showing different units in the Baikouquan ophiolitic mélange; (b) Photo showing the locations of amphibolite and metagabbro blocks in serpentinite matrix; (c) Microphtograph showing garnet amphibolite. Garnet contains abundant mineral inclusions, crossed-polarized light; (d) BSE image showing mineral inclusions in garnet. Amp - amphibole, Ap - apatite, Grt - garnet, ilm - ilmenite, Ru- rutile, $\mathrm{Zr}$ - zircon; (e) Compositional space for garnet in the Baikouquan ophiolitic mélange. 


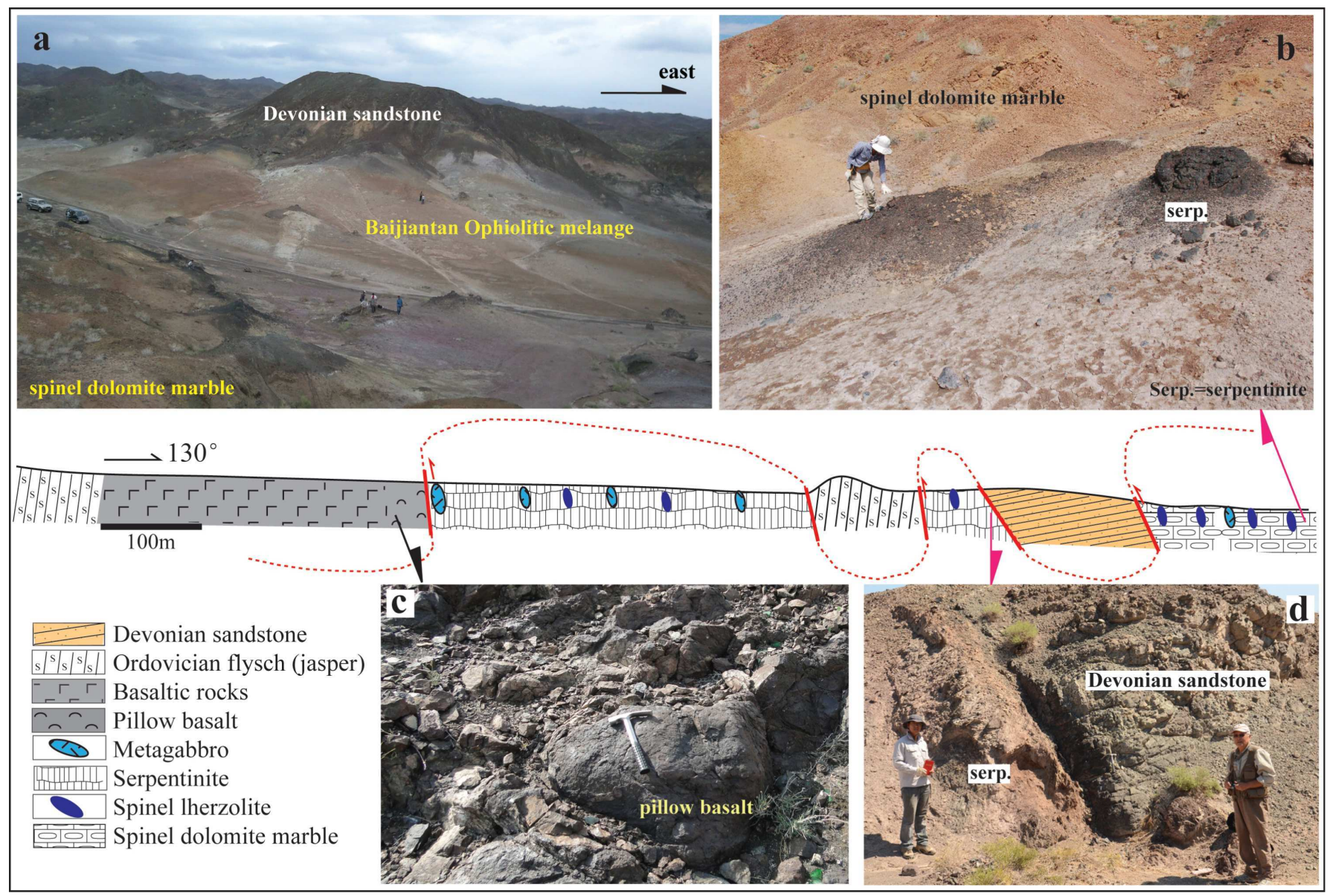

Figure 9. Geological section and photos showing the Baijiantan ophiolitic mélange. Dashed line indicates possible positions of the flysch, Devonian sedimentary strata and the eroded parts of the ophiolitic mélange. (a) Devonian sandstone lying on ophiolitic mélange, watching to NW-direction; (b) Serpentinite blocks (relics) in spinel dolomite marble; (c) Pillow basalt; (d) A fault boundary between serpentinite and Devonian sandstone.
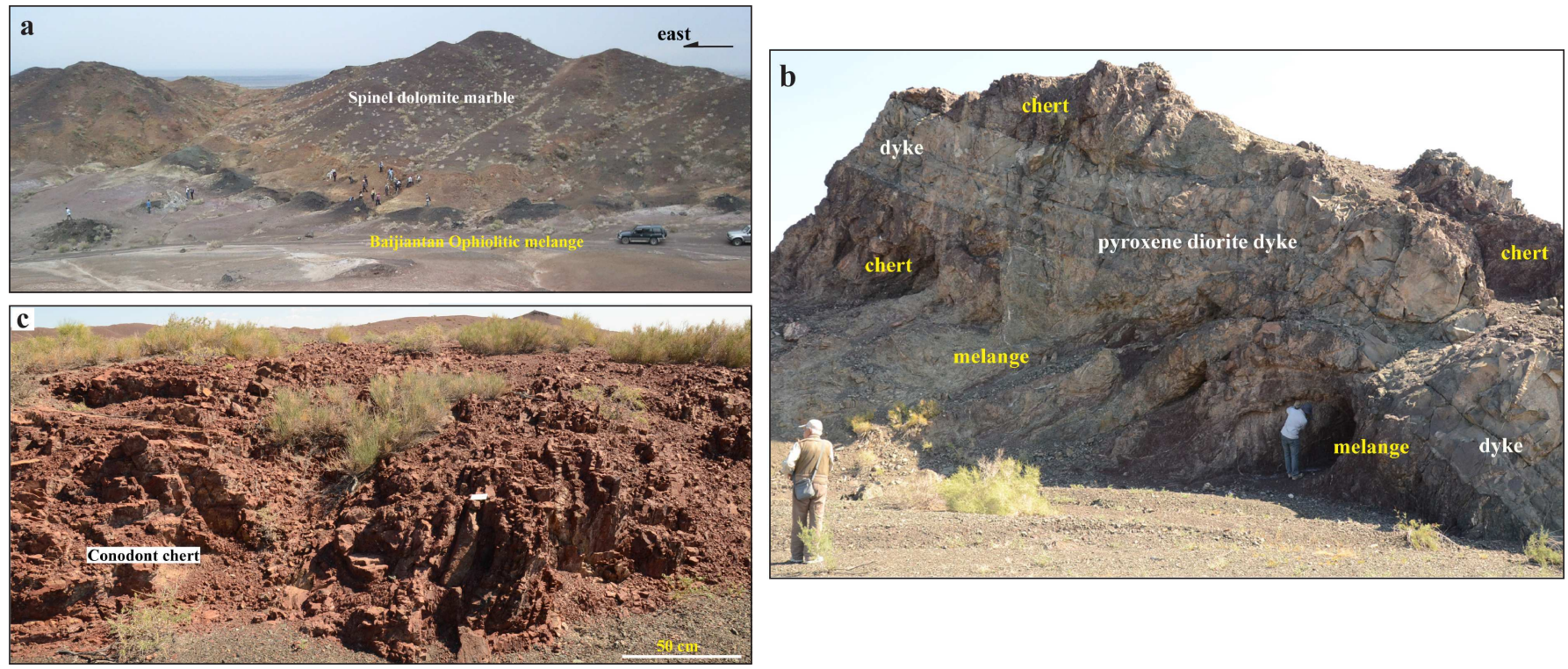

Figure 10. (a) A hill of spinel dolomite marble in the Baijiantan ophiolitic mélange, the dark-colored blocks are mainly serpentinite, some of them are metagabbro; (b) A Permian pyroxene diorite dyke intruded into deformed chert; (c) Conodont chert (Jasper) shows strong deformation. 

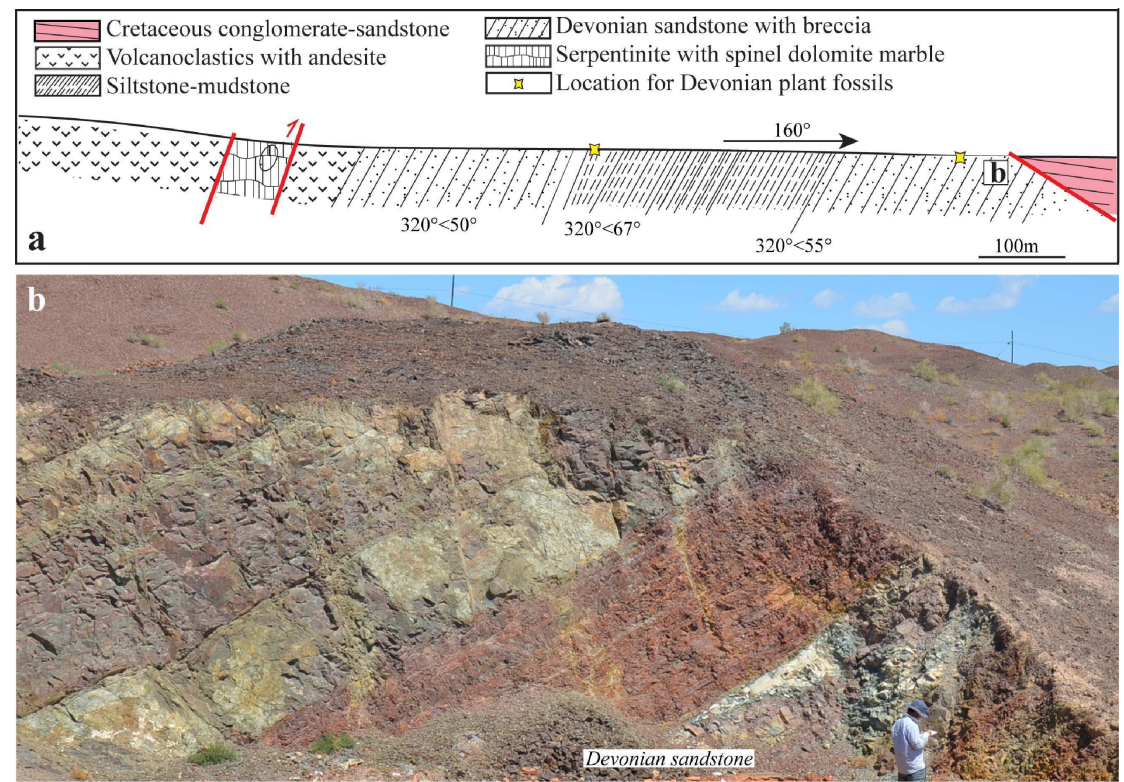

c

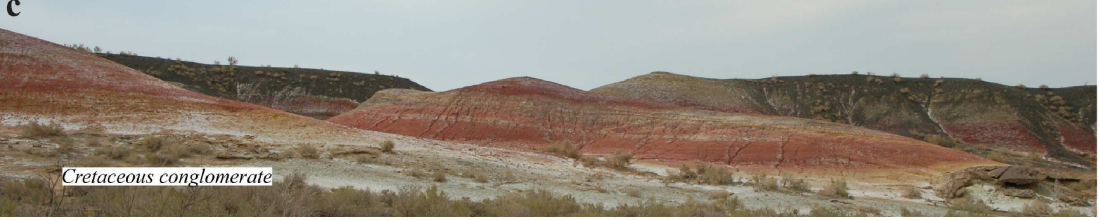

Figure 11. (a) Geological section showing the relation among blocks of serpentinite with spinel dolomite marble, Devonian sandstone and volcanoclastics with andesite, which were covered by Cretaceous conglomerate unconformably in the Baijiantan region. (b) Photo showing Devonian sandstone; (c) Photo showing Cretaceous conglomerate and sandstone.

Paleo-Ocean occurred before the Late Ordovician. The Devonian sandstone, containing plant fossils (Wang, 2008; Ma et al., 2011), did not undergo deformation while serpentinite and chert had been strongly deformed.

Figure 11 displays a geological section showing the relationship among units in ophiolitic mélange, the Devonian sandstone and the
Cretaceous conglomerate. The volcanoclasics and andesite continuously lie on the Devonian sandstone. These units contact with serpentinite via fault. Serpentinite and spinel dolomite marble occurring along fault only, which suggests a nappe structure. Cretaceous conglomerate and sandstone lie on the Devonian sandstone unconformably.

\section{U-Pb ages for post-ophiolitic magmatic rocks}

Ophiolitic mélanges and flysch in both the Darbut-Sartohay and Baijiantan- Baikouquan belts were covered by the Devonian conglomeratesandstone and Early Carboniferous volcanosedimentary rocks (see Figs. 3d-e, 9a). Andesite and volcanoclastics lie on the Devonian sandstone continuously (see Fig. 11a). The Early Carboniferous volcanoclastics with basalt and andesite are widespread in western Junggar (An and Zhu, 2007, 2009) as well as in the west Tianshan (see Zhu et al., 2009). Zircons separated from andesite in the Hatu region with typical igneous cathodoluminescence patterns have been measured by SHRIMP (see Table 1 and Figs. 12a-b). Their Th/U ratios range between 0.51 and 1.07 with apparent $\mathrm{U}-\mathrm{Pb}$ ages of $330 \sim 341 \mathrm{Ma}$. All these SHRIMP data fall on $\mathrm{U}-\mathrm{Pb}$ concordia and give an average age of $335.2 \pm 2.9 \mathrm{Ma}(\mathrm{MSWD}=1.3, \mathrm{n}=10)$. Zircons separated from tuff in the Baobei region show typical magmatic zonation with $\mathrm{Th} / \mathrm{U}$ ratios of $0.43-1.15$ and apparent $\mathrm{U}-\mathrm{Pb}$ ages of $322 \sim 349 \mathrm{Ma}$. These data fall on U-Pb concordia and give an average age of $328.1 \pm 1.8 \mathrm{Ma}$ (MSWD = 1.6, $\mathrm{n}=13$, Figs. 12c-d).

These U-Pb ages (335Ma, 328Ma), representing magma eruption in the Hatu and Baobei regions, are consistent with zircon $\mathrm{U}-\mathrm{Pb}$ ages for volcanic rocks in the Baogutu region (328 - 343Ma, An and Zhu,
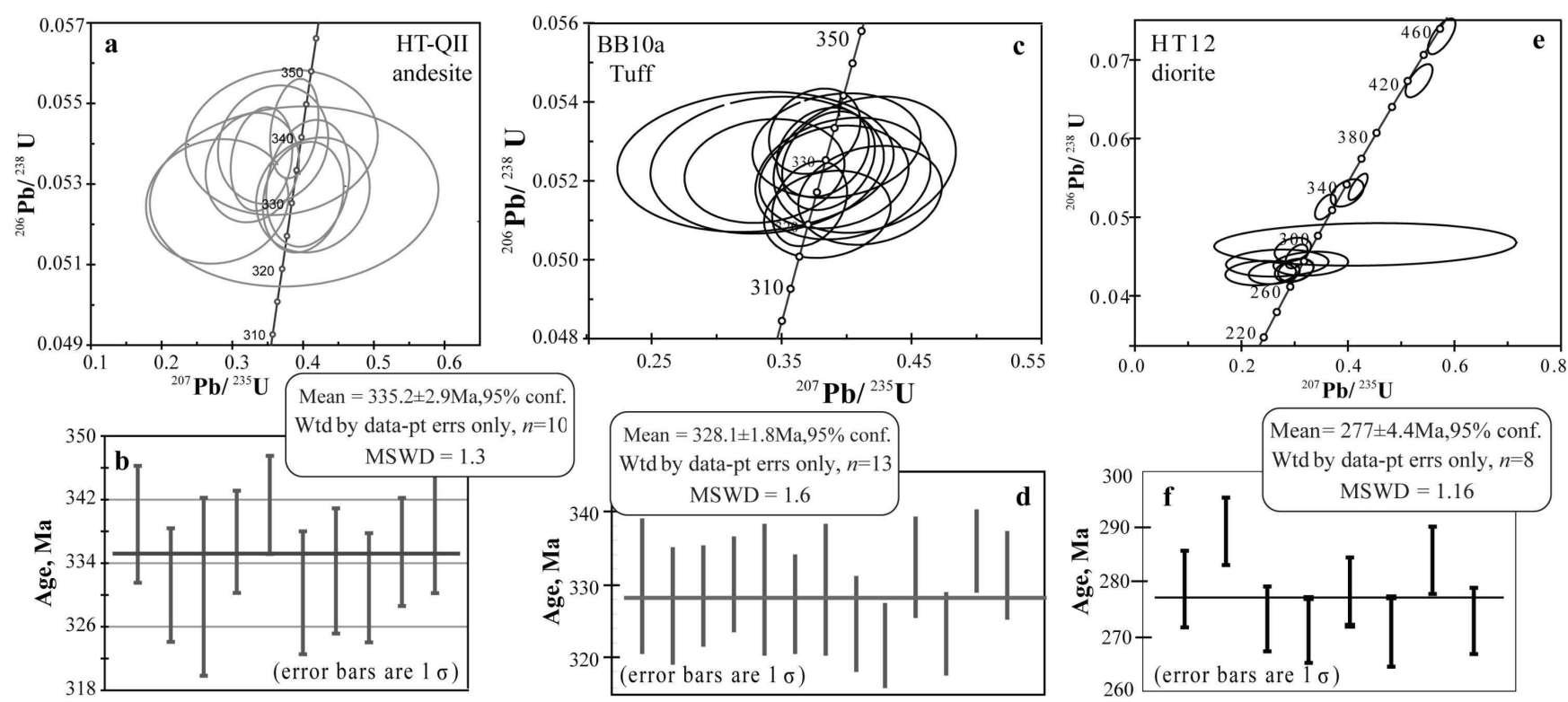

Figure 12. SHRIMP dating results for zircons separated from andesite in the Hatu (a-b), from tuff in the Baobei (c-d), and from diorite dyke intruded into Early Carboniferous volcanoclastics (e-f). 

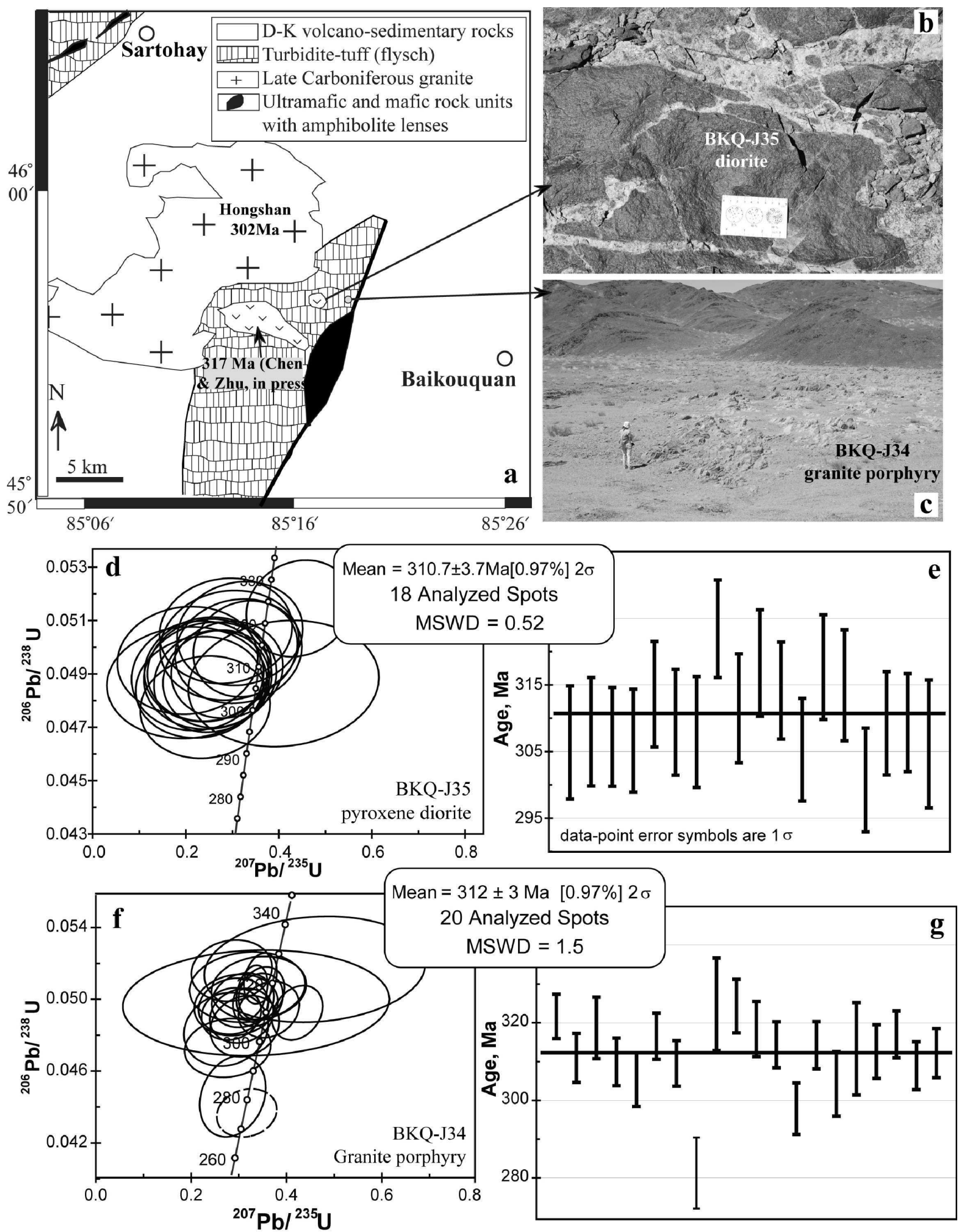

Figure 13. (a) Simplified geological map of the Baikouquan region. The large granite body (Hongshan pluton) has been dated to be $302 \mathrm{Ma}$ by Han et al. (2010), and a small diorite pluton has been dated to be 317Ma by Chen and Zhu (in press); (b) Photo showing an outcrop of diorite intruded into siltstone - chert - tuff, and has been cut by granite veins; (c) Photo showing an outcrop of granite porphyry intruded into siltstone-tuff; d-g) Zircon SHRIMP dating results for diorite (d-e) and for granite porphyry (f-g). 
2009; Guo et al., 2010). Except for large granite bodies ( 300Ma, Han et al., 2006), small granitic to diorite plutons and various dykes intruded into the Devonian to Early Carboniferous volcanosedimentary units (Han et al., 2010; Liu et al., 2009; Wei et al., 2011). Zircons separated from a diorite dyke in the Hatu region have highly variable U-Pb ages (454 - 271Ma, see Fig. 12e) with $\mathrm{Th} / \mathrm{U}$ ratios of 0.46 - 0.70. All these SHRIMP data fall on U-Pb concordia, and the apparently younger $\mathrm{U}-\mathrm{Pb}$ age values give an average age of 277.0 $\pm 4.4 \mathrm{Ma}$ (MSWD $=1.16, \mathrm{n}=8$, see Figs. 12e-f). Two apparently older ages (454.2 Ma, 420.2Ma, see Table 1 for spot -2.1 and -3.1) are similar to the $\mathrm{U}-\mathrm{Pb}$ age of metagabbro in ophiolitic mélange (see Fig. 6).

Magmatic plutons generally intruded into the Early Carboniferous volcano-sedimentary strata, while some of them intruded into the flysch accompanying ophiolitic mélange. Here we show two small intrusive bodies in the Baikouquan ophiolitic mélange (Figs. 13a-c). A small diorite pluton, intruded into tuff-siltstone, was cut through by granite veins (see Fig. 13b). Zircons in this diorite show typical igneous zonation pattern in cathodoluminescene image. The apparent $\mathrm{U}-\mathrm{Pb}$ ages vary from $300.7 \mathrm{Ma}$ to $323.4 \mathrm{Ma}$ with $\mathrm{Th} / \mathrm{U}$ ratios of 0.43 0.63 . All 18 analyses fall on $\mathrm{U}-\mathrm{Pb}$ concordia and give an average age of $310.7 \pm 3.7 \mathrm{Ma}$ (MSWD $=0.52$, see Figs. $13 \mathrm{~d}-\mathrm{e}$ ). The zircons separated from a small granite porphyry (see Fig. 13c) have apparent $\mathrm{U}-\mathrm{Pb}$ ages of $275.5-325 \mathrm{Ma}$ with $\mathrm{Th} / \mathrm{U}$ ratios of $0.39-0.94$. Except one analysis (spot -10.1), other 20 analyses fall on U-Pb concordia and give an average age of $312 \pm 3 \mathrm{Ma}$ (MSWD $=1.5$, see Figs. 13fg). The younger age (275.5 Ma) might relate with late-stage fluid alteration widespread in this region.

\section{Discussion}

New geological data suggest that the western Junggar mainly formed during the closing of the Cambrian-Ordovician Ocean. The relics of the Early Paleozoic oceanic crust (ophiolitic mélanges) were covered by Devonian sandstone and Early Carboniferous volcanoclastics with lavas. The latter are widespread in the western Junggar and were previously mis-understood as arc system in geological literature. Geological evidences for the closure of the CAPO at pre-Devonian (see Fig. 3 in the Sartohay region and Fig. 9a in the Baijiantan region) are consistent with the U-Pb age of metagabbro in the Darbut ophiolitic mélange (426 Ma, see Fig. 6).

The new geological observations are critical for Paleoenvironmental reconstruction of the western Junggar. As a major part of the Kazakhstan-Junggar plate, the western Junggar was formed during the Ordovician to Silurian period. Individual lithologic formations are well exposed in the western Junggar, where the Lower Devonian marine deposition is continuous with the Upper Silurian (Ma et al., 2011), and the Middle Devonian terrestrial deposition contains abundant plant fossils (Wang, 2008). However, the occurrence of Silurian strata is rare. In most cases, the Devonian to Early Carboniferous molasse covered on the strongly deformed ophiolitic mélanges and flysch. The post-orogenic intermediate to felsic magmatism intruded into molasse and controlled the coppergold porphyry and lode gold deposits (Zhu et al., 2013).

The time scale for geological evolution could be simply illustrated with $\mathrm{U}-\mathrm{Pb}$ age distribution pattern for magmatic rocks in the western Junggar (Fig. 14a). Most U-Pb age values vary between 300Ma and $360 \mathrm{Ma}$ with one peak at $318 \mathrm{Ma}$ and another peak at $345 \mathrm{Ma}$. Some old age data (420 - 460Ma) belong to Silurian period. The SHRIMP
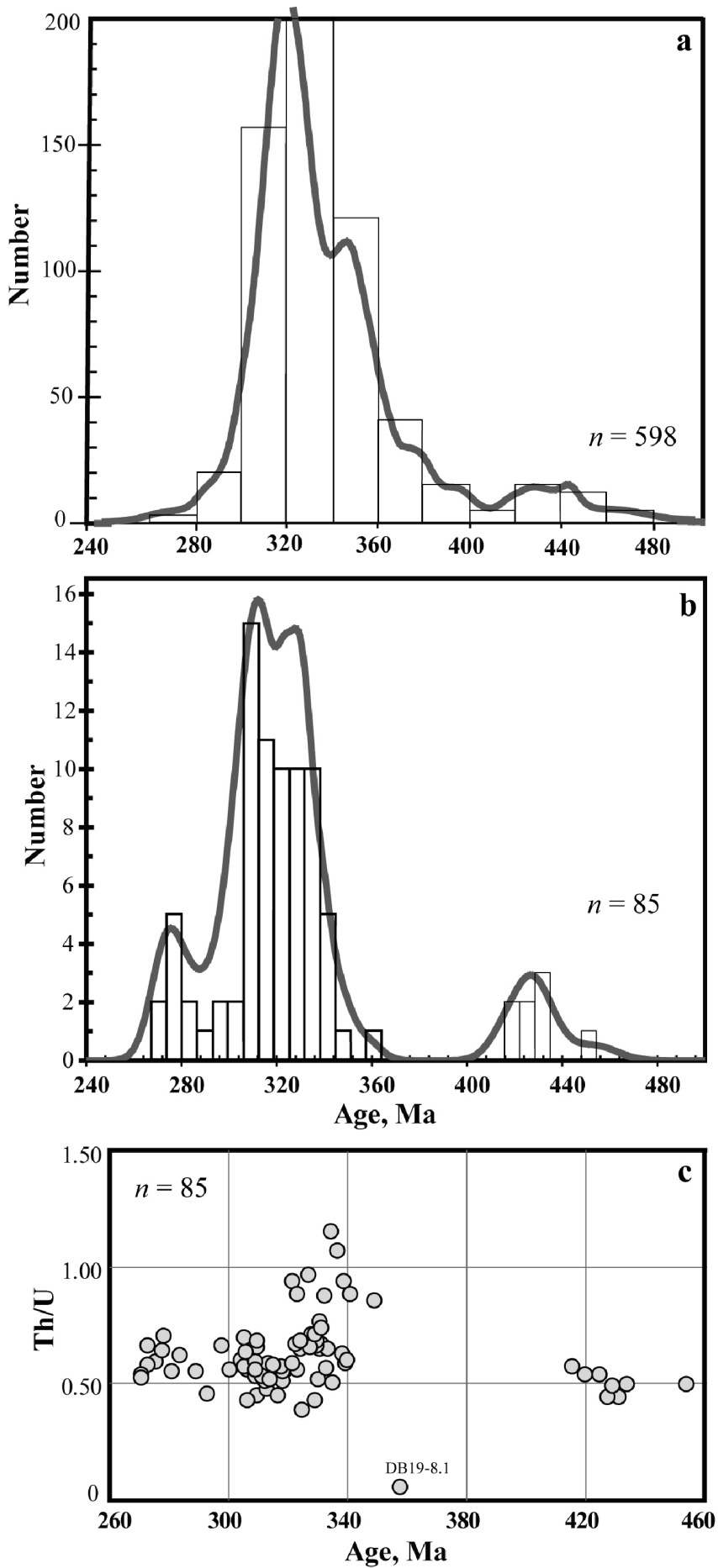

Figure 14. (a) Histogram of $U-P b$ age distribution for igneous rocks in the western Junggar (data from references: Xu et al., 2006; Han et al., 2006, 2010; An and Zhu, 2009; Gu et al., 2009; Guo et al., 2010; Chen et al., 2010; Wei et al., 2011; Chen and Zhu, in press); (b) Histogram of zircon SHRIMP ages for igneous rocks studied here (data are listed in Table 1); (c) The correlations for zircon $U$-Pb ages and their $T h / U$ ratios.

data-set presented in Table 1 is shown in Fig. 14b, from which four age-peaks could be identified. The peak corresponding to the middle Silurian zircons separated from metagabbro in ophiolitic mélange implies that the Paleo-Ocean floor spreading did not stop until the middle Silurian. One peak at Early Carboniferous is formed by zircons 
separated from volcanoclastics lying on flysch, while the peak at Late Carboniferous corresponds to zircons in magmatic plutons intruded into flysch and volcano-sedimentary units. The Early Permian zircons are from dykes. All these zircons have high $\mathrm{Th} / \mathrm{U}$ ratios $(>0.4)$ except only one $(<0.1$, see Fig. $14 \mathrm{c})$, which might be a mixture between igneous zircon and its metamorphic rim. Although the $\mathrm{U}-\mathrm{Pb}$ age value corresponding to such mixed zircon may not have definite geological significance (Zhu, 2011), the observation of such metamorphic rim after igneous zircon is consistent with the metamorphism developed in the studied metagabbro. This is also consistent with the ductileshearing deformation recorded in magnesitite (see Fig. 3c). The Devonian to Early Carboniferous sandstone and volcano-sedimentary units lying on the ophiolitic mélange did not undergo any metamorphism. This constrains the upper time limit for ductileshearing deformation in the western Junggar, and implies that the emplacement of ophiolitic mélanges in the western Junggar happened pre-Devonian.

\section{Conclusion}

The newly-compiled geological map shows that the western Junggar mainly formed during the closing of the Cambrian-Ordovician Ocean. The relics of Early Paleozoic oceanic crust were mainly covered by Devonian sandstone to Early Carboniferous volcanoclastics with andesite and basalt. The latter are widespread and were previously misunderstood as arc system related to the closure of the CAPO. The evidences for the Devonian closing of the Paleoocean as represented by the Darbut-Sartohay and BaijiantanBaikouquan ophiolitic mélanges are apparent in field observations and isotopic ages. The newly compiled geological map shows two different geological layers: the Early Paleozoic metamorphic terrane mainly consists of ophiolitic mélanges and flysch (the Baobei-HLS, Darbut-Sartohay, and Baijiantan-Baikouquan belts), which have undergone strong ductile-shearing deformation and metamorphism, and the Late Paleozoic sandstone and volcano-sedimentary units lying on the ophiolitic mélanges and flysch. Both of these geological layers have been intruded by granite to diorite during Late Carboniferous to Early Permian.

\section{Acknowledgements}

We would like to thank the anonymous Episodes reviewer who provided critical comments and suggestions. Our thanks are also due to the International S\&T Cooperation Program of China (Grant 2010DFB23390) and Natural Science Foundation of China (41072041, 41121062) for financial support. Discussion with Prof. Guoqi He and Baofu Han helped us to complete this paper and, are, therefore gratefully acknowledged.

\section{References}

An, F. and Zhu, Y.F., 2007. Studies on geology and geochemistry of alterationtype ore in Hatu gold deposit (western Junggar), Xinjiang, NW China: Mineral Deposits, v. 26, pp. 621-633 (in Chinese with English abstract).

An, F. and Zhu Y.F., 2009. SHRIMP U-Pb zircon ages of tuff in Baogutu formation and their geological significances: Acta Petrologica Sinica, v. 25, pp. 1437-1445 (in Chinese with English abstract).

Bai, W.J., Yang, J.S., Zhou, M.F. and Hu, X.F., 1995. Tectonic evolution of different dating ophiolites in western Junggar, Xinjiang: Acta Petrologica Sinica, v. 11 (suppl), pp. 62-72 (in Chinese with English abstract).
BGMRXUA, 1993. Regional geology of Xinjiang Uygur Autonomous Region: Beijing: Geol. Pub. House (in Chinese).

Chen, B. and Zhu, Y.F., 2008. Petrology of ultramafic rock in Darbut ophiolite (Xinjiang), evidence from Cr-spinel: Earth Science Frontiers, v. 15, pp. 312 - 322 (in Chinese with English abstract).

Chen, B. and Zhu, Y.F. Petrology, geochemistry and U-Pb chronology of the post-ophiolitic gabbro-diorite in western Junggar. Acta Petrologica Sinica, (in press).

Choulet, F, Faure, M, Cluzel, D, Chen, Y., Lin, W. and Wang, B., 2012. From oblique accretion to transpression in the evolution of the Altaid collage: New insights from West Junggar, northwestern China: Gondwana Research, v. 21, pp. 530-547.

Coleman, R.G, Lee, D.E., Beatty, L.B. and Brannock, W.W., 1965. Eclogites and eclogites: their difference and similarilities: Bull. Geol. Soc. America, v. 76, pp. 483-580.

Feng, Y.M., 1986. Genetic environments and original types of ophiolites in west Junggar: Bull. Xi'an Inst. Geol. Min. Res., Chinese Acad. Geol. Sci., v. 13, pp. 37-45 (in Chinese with English Abstract).

Gu, P., Li, Y., Zhang, B., Tong, L. and Wang, J., 2009. LA-ICP-MS zircon $\mathrm{U}-\mathrm{Pb}$ dating of gabbro in the Darbut ophiolite, western Junggar, China: Acta Petrologica Sinica, v. 25, pp. 1364-1372 (in Chinese with English abstract).

Guo, L.S., Liu, Y.L., Wang, Z.H., Song, D., Xu, F.J. and Su, L., 2010. The zircon U-Pb LA-ICP-MS geochronology of volcanic rocks in Baogutu areas, western Junggar: Acta Petrologica Sinica, v. 26, pp. 471-477 (in Chinese with English abstract).

Han, B.F., Ji, J.Q. and Song, B., 2006. Late paleozoic vertical growth of continental crust around the Junggar Basin, Xinjiang, China (part I): Timing of post-collisional plutonism: Acta Petrologica Sinica, v. 22, pp. 1077-1086 (in Chinese with English Abstract).

Han, B.F., Guo, Z.J. and He, G.Q., 2010. Timing of major suture zones in North Xinjiang, China: Constraints from stitching plutons: Acta Petrologica Sinica, v. 26, pp. 2233-2246 (in Chinese with English Abstract).

Han, B.F., He, G.Q., Wang, X.C. and Guo, Z.J., 2011. Late Carboniferous collision between the Tarim and Kazakhstan-Yili terranes in the western segment of the South Tian Shan Orogen, Central Asia, and implications for the Northern Xinjiang, western China. Earth-Science Reviews, v. 109, pp. 74-93.

He, G.Q., Chen, S.D., Xu, X., Li, J.Y. and Hao, J. 2004. An introduction to the explanatory text of the map of tectonics of Xinjiang and its neighboring area (1: 250000, in Chinese with English abstract): Beijing, Geological Publishing House.

He, G.Q., Liu, J.B., Zhang, Y.Q. and Xu, X., 2007. Karamay ophioliic mélange formed during Early Paleozoic in western Junggar basin: Acta Petrologica Sinica, v. 23, pp. 1573-1576 (in Chinese with English abstract).

Heinhorst, J., Lehmann, B., Ermolov, P., Serykh, V. and Zhurutin, S., 2000. Paleozoic crustal growth and metallogeny of Central Asia: evidence from magmatic-hydrothermal ore systems of Central Kazakhstan: Tectonophysics, v. 328, pp. 69-87.

Kwon, S.T., Tilton, G.R., Coleman, R.G. and Feng, Y.M., 1989. Isotopic studies bearing on the tectonics of west Junggar region, Xinjiang, China. Tectonic, v. 8, pp. 719-727.

Kröner, A., Hegner, E., Lehmann, B., Heinhorst, J., Wingate, M.T.D., Liu, D.Y. and Ermelov, P., 2008. Palaeozoic arc magmatism in the Central Asian Orogenic Belt of Kazakhstan: SHRIMP zircon ages and wholerock Nd isotopic systematics: Journal of Asian Earth Sciences, v. 32, pp. $118-130$.

Kurchavov, A.M., Grankin, M.S., Malchenko, E.G., Khamzin, B.S. and Zhukovskii, V.I., 2002. Metallogenic zonality of the Devonian volcanoplutonic belt in Central Kazakhstan: Geology of Ore Deposits, v. 44, pp. 18-25 (in Russia).

Lei, M., Zhao, Z.D. and Hou, Q.Y., 2008. Geochemical and Sr-Nd-Pb isotopic characteristics of the Dalabute ophiolite, Xinjiang: Comparison between the Paleo-Asian ocean and the Tethyan mantle domains. Acta Petrologica Sinica, v. 24, pp. 661-672 (in Chinese with English abstract). 
Liu, Y.L., Guo, L.S., and Liu, Y.D., 2009. Geochronology of Baogutu porphyry copper deposit in Western Junggar area, Xinjiang of China: Science in China Series D: Earth Sciences, v. 52, pp. 1543-1549.

Ma, X.P., Zong, P. and Sun, Y.L., 2011. The Devonian (Famennian) sequence in the western Junggar area, north Xinjiang, China. Subcommission on Devonian Stratigraphy: SDS Newsletter, no 26, pp. 44-49.

Qiu, T. and Zhu, Y.F., 2012. Identification of a ductile shear zone in quartzmagnesitite and its controlling on gold deposits in the Sartohay region, western Junggar, Xinjiang: Acta Petrologica Sinica, v. 28, pp. 2250-2256 (in Chinese with English Abstract).

Tang, J.J. and Zhu, Y.F. 2010. Study on Fe-Ni-As-S assemblages and platinumgroup minerals in Sartohay chromite deposit, Xinjiang, China: Acta Petrologica Sinica, v. 26, pp. 2264-2274 (in Chinese with English Abstract).

Wang, D.M., 2008. A new iridopteridalean plant from the Middle Devonian of northwest China: Int. J. Plant Sci., v. 169, pp. 1100-1115.

Wei, S.N., Cheng, J.F., Yu, D.B. and Zhu,Y.F., 2011. Petrology and SHRIMP zircon ages of intrusive body III in Baogutu area, Xinjiang: Earth Science Frontiers, v. 18, pp. 212-222 (in Chinese with English Abstract).

Wei, W., Dong, X., Zeng, H. and Gao, J., 1987. The Geological Characters and Genesis of Ultrabasic Rock Mass and Chromite Deposit in Sartohay of Xinjiang, China: Bulletin of Xi' an Institute of Geology, Chinese Academy of Geological Scineces, v. 16, pp. 57-145 (in Chinese).

Williams, I.S., 1998. U-Th-Pb geochronology by ion microprobe, in: McKibben M.A., Shanks III W.C., Ridley, W.I. (eds.), Applications of microanalytical techniques to understanding mineralization processes: Reviews in Economic Geology, v. 7, pp. 1-35.

Wilhem, C., Windley, B.F. and Stampfli, G.M., 2012. The Altaids of Central Asia: A tectonic and evolutionary innovative review: Earth-Science Reviews, v. 113, pp. 303-341.

Windley, B.F., Alexeiev, D. and Xiao, W.J., 2007. Tectonic models for accretion of the central Asian orogenic belt: Journal of the Geological Society of London, v. 164, pp. 31-47.

Xiao, W.J., Han, C.M. and Yuan, C., 2008. Middle Cambrian to Permian subduction-related accretionary orogenesis of Northern Xinjiang, NW China: implications for the tectonic evolution of central Asia: Journal of Asian Earth Sciences, v. 32, pp. 102-117.

$\mathrm{Xu}, \mathrm{X}$., He, G.Q. and Li, H.Q., 2006. Basic characteristics of the Karamay ophiolitic mélange, Xinjiang, and its zircon SHRIMP dating: Geology in China, v. 33, pp. 470-475 (in Chinese with English Abstract).

Yakubuchuk, A., 2004. Architecture and mineral deposit settings of the Altaid orogenic collage: a revised model: Journal of Asian Earth Sciences, v. 23, pp. 761-779.

Yang, G.X., Li, Y.J., Gu, P.Y., Yang, B.K., Tong, L. and Zhang, H.W., 2012. Geochronological and geochemical study of the Darbut Ophiolitic Complex in the West Junggar (NW China): Implications for petrogenesis and tectonic evolution. Gondwana Research, v. 21, pp. 1037-1049.

Yang, R.Y., Tang, H.F., Liu, C.Q., Takahashi, K., Masuda, A. and Wei, L., 2000. Geochemistry of mafic rocks from Dalabute ophiolite in West Junggar, Xinjiang, NW China. Acta Mineralogica Sinica, v. 20, pp. 363370 (in Chinese with English abstract).

Zhang, C. and Huang, X., 1992. The age and tectonic setting of ophiolites in west Junggar, Xinjiang: Geological Reviews, v. 38, pp. 509-524 (in Chinese with English abstract).

Zhang, C., Zhai, M.G., Allen, M.B., Ssunders, A.D., Wang, G.R. and Huang, X., 1993. Implications of Palaeozoic ophiolites from western Junggar, NW China, for the tectonics of central Asia. Journal of Geological Society, London, v. 150, pp. 551-561.

Zhang, L.F., 1997. ${ }^{40} \mathrm{Ar} /{ }^{39} \mathrm{Ar}$ age of blueschists in Tangbale, western Junggar, Xinjiang, and its significance: Chinese Science Bulletin, v. 42, pp. 21782181 (in Chinese).

Zhang, L.F., Sun, M. and Xu, B., 2001. Phase relations in garnet-bearing metabasites of prehnite- pumpellyite facies from the Darbut-Sartuohai ophiolite, western Junggar of Xinjiang, China. Mineral and Petrology, v. 71, pp. 67-85.

Zhao, L. and He, G., 2012. Tectonic entities connection between West Junggar
(NW China) and East Kazakhstan: Journal of Asian Earth Sciences, http:/ /dx.doi.org/10.1016/j.jseaes.2012.08.004

Zhou, L.R., Zhao, Z.C. and Zhang, J.S., 1987. The essential features of geotectonic development and magmatic evolution on the western Junggar area Xinjiang, China: Bulletin of Xi'an Institute of Geology, Chinese Academy of Geological Scineces, v. 17, pp. 3-64 (in Chinese).

Zhou, M., Robinson, P.T., Malpas, J. and Aitchison J., 2001. Melt/mantle interaction and melt evolution in the Sartohay high-Al chromite deposits of the Dalabute ophiolite (NW China): Journal of Asian Earth Sciences, v. 19, pp. 517-534.

Zhu, B., Wang, L. and Wang, L., 1987. Paleozoic Era ophiolite of southwest part in western Junggar, Xinjiang, China: Bulletin of Xi' an Institute of Geology, Chinese Academy of Geological Scineces, v. 17, pp. 3-64 (in Chinese).

Zhu, Y.F., 2011. Zircon U-Pb and muscovite ${ }^{40} \mathrm{Ar} /{ }^{39} \mathrm{Ar}$ geochronology of the gold-bearing Tianger mylonitized granite, Xinjiang, northwest China: Implications for radiometric dating of mylonitized magmatic rocks: Ore Geology Reviews, v. 40, pp. 108-121.

Zhu, Y.F. and Ogasawara, Y., 2002. Carbon recycled into the deep Earth: Evidenced by dolomite dissociation in subduction-zone rocks: Geology, v. 30 , pp. $947-950$.

Zhu, Y.F. and Xu, X., 2006. The discovery of Early Ordovician ophiolite mélange in Taerbahatai Mts., Xinjiang, NW China: Acta Petrologica Sinica, v. 22, pp. 2833-2842 (in Chinese with English Abstract).

Zhu, Y.F., Xu, X., Wei, S.N., Song, B. and Guo, X., 2007. Geochemistry and tectonic significance of OIB-type pillow basalts in western Mts. of Karamay city (western Junggar), NW China: Acta Petrologica Sinica, v. 23(7), pp. 1739-1748 (in Chinese with English abstract).

Zhu, Y.F., Xu, X., Chen, B. and Xue, X.Y., 2008. Dolomite marble and garnet amphibolite in the ophiolitic mélange in western Junggar: Relics of the Early Paleozoic oceanic crust and its deep subduction: Acta Petrologica Sinica, v. 24, pp. 2767-2777 (in Chinese with English abstract).

Zhu, Y.F., Guo, X., Song, B., Zhang, L.F. and Gu, L.B., 2009. Petrology, $\mathrm{Sr}-\mathrm{Nd}-\mathrm{Hf}$ isotopic geochemistry and zircon chronology of the Late Palaeozoic volcanic rocks in the southwestern Tianshan Mountains, Xinjiang, NW China. Journal of the Geological Society, London, v. 166, pp. 1085-1099

Zhu, Y.F., Yan, Q.M., Ma, H.D. and Lehmann, B., 2011. Recent advances in geology and exploration in the Balkash-western Junggar region (Kazakhstan and Xinjiang, China): Report on the "International workshop on the large Balkash-western Junggar copper-gold province", Karamay, Xinjiang, China, 22-27 August, 2011. Episodes, v. 34(3), pp. 208-211.

Zhu, Y.F., An, F., Xu, CY. and others, 2013. Geology and Au-Cu deposits in the Hatu and its adjacent region (Xinjiang) : evolution and prospecting model: Beijing, Geological Publishing House, pp. 1-161.

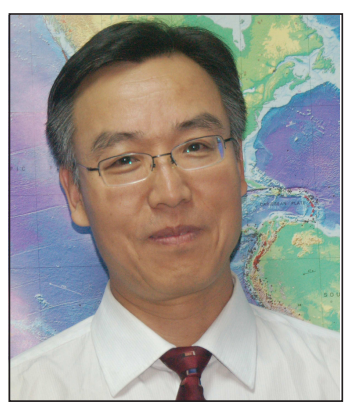

Yongfeng Z $\mathbf{h u}$, Professor at the Peking University (China), got his $\mathrm{PhD}$ in Petrology and Mineralogy from the Moscow State University (Russia, 1993). His research interests are geodynamics and metallogeny of ore deposits in central Asia. 